\title{
Modeling Constituent Redistribution in U-Pu-Zr Metallic Fuel Using the Advanced Fuel Performance Code BISON
}

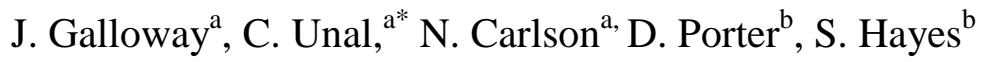 \\ ${ }^{a}$ Los Alamos National Laboratory, P.O. Box 1663, Los Alamos, NM 87545, United States \\ ${ }^{\mathrm{b}}$ Idaho National Laboratory, 2525 N. Fremont Avenue, P.O. Box 1625, Idaho Falls, ID 83415 \\ *Corresponding author: Los Alamos National Laboratory, P.O. Box 1663, Mail Stop F606, Los Alamos, \\ NM 87545, USA. Tel.: +1 505665 2539; fax: +1 505665 5204; E-mail address: cu@lanl.gov.
}

\begin{abstract}
An improved robust formulation for constituent distribution in metallic nuclear fuels is developed and implemented into the advanced fuel performance framework BISON. The coupled thermal diffusion equations are solved simultaneously to reanalyze the constituent redistribution in post irradiation data from fuel tests performed in Experimental Breeder Reactor II (EBR-II). Deficiencies observed in previously published formulation and numerical implementations are also improved. The present model corrects an inconsistency between the enthalpies of solution and the solubility limit curves of the phase diagram while also adding an artificial diffusion term when in the 2-phase regime that stabilizes the standard Galerkin Finite Element (FE) method used by BISON. An additional improvement is in the formulation of zirconium flux as it relates to the Soret term. With these new modifications, phase dependent diffusion coefficients are revaluated and compared with the previously recommended values.

The model validation included testing against experimental data from fuel pins T179, DP16 and T459, irradiated in EBR-II. A series of viable material properties for U-Pu-Zr based materials was determined through a sensitivity study, which resulted in three cases with differing parameters that showed strong agreement with one set of experimental data, rod T179.

Subsequently a full-scale simulation of T179 was performed to reduce uncertainties, particularly relating to the temperature boundary condition for the fuel. In addition a new thermal conductivity model combining all available data covering 0 to $100 \%$ zirconium concentration and a zirconium concentration dependent linear heat rate solution derived from Monte Carlo NParticle (MCNP) simulations were developed. An iterative calibration process was applied to obtain optimized diffusion coefficients for $\mathrm{U}-\mathrm{Pu}-\mathrm{Zr}$ metallic fuels. Optimized diffusion coefficients suggest relative improvements in comparison to previous reported values. The most influential or uncertain phase is found to be the gamma phase, followed by alpha phase, and thirdly the beta phase; indicating separate effect testing should concentrate on these phases.
\end{abstract}

Keywords: Nuclear, Fuels, Metallic, Constituent, Species, Diffusion, Redistribution, Bison

\author{
Abbreviations \\ EBR-II $\quad$ Experimental Breeder Reactor - II
}




$\begin{array}{ll}\text { FE } & \text { Galerkin Finite Element (FE) } \\ \text { LWR } & \text { Light water reactor } \\ \text { M\&S } & \text { Modeling and simulation } \\ \text { MCNP } & \text { Monte Carlo N-Particle } \\ \text { US } & \text { United States }\end{array}$

\section{Introduction}

In the past, both metallic and oxide nuclear fuels were tested in fast reactor programs and performed well (Walters, 1999). The recent nuclear accident at Fukushima shifted the advanced fuels research focus to the development of accident tolerant fuels for light water as well as small modular reactor applications. The use of metallic fuels in these new nuclear technology concepts has been gaining renewed attention.

Metallic fuels are attractive since they have higher thermal conductivity with a highly conductive gap (typically sodium) that enables the fuel to operate at lower temperatures with reduced stored energy. Additional benefits of metallic fuels include a more favorable neutron economy, higher fuel densities, and easier fabrication and reprocessing.

Metallic fuels tested previously typically consisted of a binary alloy of uranium and zirconium, or a ternary alloy of uranium, plutonium and zirconium. The addition of $\sim 10 \%$ by weight zirconium to a uranium-plutonium alloy raises the solidus temperature of the fuel and prevents fuel cladding inter diffusion at temperatures typical of steady operating conditions (Walters, 1999). The addition of americium, neptunium, and curium to the traditional metallic fuel is one aspect considered for closing the fuel cycle. New metallic fuel concepts also include the use of uranium-molybdenum and uranium-palladium-zirconium based fuels.

Generally speaking, the thermo-mechanical behavior of a nuclear fuel pellet or rod involves a complex system of interdependent processes as a result of the high thermal-power densities and irradiation effects. All of the physics are driven by processes occurring at the microstructure level (i.e., at the grain or subgrain scale). The migration of porosity, fuel constituents, and fission products causes fuel restructuring in metal fuels. Irradiation induced effects such as fission product generation, as well as chemical interactions, change the material properties of the fuel and cladding. Microstructural characteristics and their irradiation induced evolution can also influence material properties. Fuel swelling, a result of both gaseous and solid fission product generation, and atomic displacements due to the neutron flux, have significant impacts on dimensional changes and mechanical properties of the materials. Swelled fuel causes mechanical interaction between fuel and cladding that can produce stresses and deformation in addition to the stress caused by internal pressure in the fuel. The nuclear (heat and irradiation) source drives many of these processes, which can vary greatly in thermal reactors. In addition, the chemical interactions between cladding and coolant significantly affect the heat transfer coefficient and 
mechanical strength of the cladding; these interactions can be driven by both local (nucleate boiling) and global (soluble metals) coolant flow conditions.

The behavior of metallic fuel undergoes various stages during irradiation. A detailed description of the metallic fuel behavior under irradiation is available in previously published papers (Walters, 1999), (Carmack et al., 2009), Hoffman et al., 1996), (Karahan and Buongiorno, 2009). One important key design and modeling issue with metallic fuels has been found to be the constituent distribution; specifically zirconium redistribution (Walters, 1999), (Carmack et al., 2009), (Hoffman et al., 1996), (Karahan and Buongiorno, 2009), (Hofmann et al, 1996), (Kim et al, 2004, 2005, 2006, 2009), (Ishida et al., 1993). Fuel constituent migration affects the fuel slug material properties, such as solidus and liquidus temperatures, thermal conductivity, along with mechanical properties such as the modulus of elasticity, fuel cladding eutectic reaction rate, and radial power density profile.

Zirconium redistribution in $\mathrm{U}-\mathrm{Zr}$ and $\mathrm{U}-\mathrm{Pu}-\mathrm{Zr}$ based fuels is important for both fuel integrity and thermal limits. As zirconium redistributes, the uranium moves in the opposite direction and what was once a uniform isotopic concentration across the fuel rod becomes concentrated in zirconium, primarily at rod center and rod edge. This preferential accumulation at rod edge and rod center, with a corresponding decrease in the central region can affect local thermal limits (margin to melting temperature for example), radial power peaking, with potential enhances to lanthanide and actinide migration, all due to a significantly varying radial isotopic composition. A strong understanding of this process along with a robust method for predicting zirconium concentrations is vital to advanced metallic fuel designs, particularly those designs with minor actinide (MA) loadings, lanthanide loadings and new proposed metallic alloys.

The prediction of zirconium redistribution is difficult for several reasons. First, fuel behavior under irradiation is a multi-physics, multi-scale problem that requires the coupling of neutronic solutions due to uranium migration which induces radial power peaking. Correspondingly, burnup effects in the fuel cause changes in both power and the thermo-mechanical-diffusion fuel behavior. Additionally there are thermal considerations primarily related to thermal conductivity; as zirconium migrates, the thermal conductivity changes inversely with zirconium concentration - an increase in zirconium concentration causing a decrease in thermal conductivity and vice-versa. Fuel burnup increases porosity in the fuel, which degrades thermal conductivity. However, near the rod edge liquid sodium infiltration due to fuel cracking can occur, causing some recovery of the degraded conductivity. This porosity itself appears to be phase-dependent, particularly in the beta phase region of the fuel where porosity appears to be at a minimum and thermal conductivity degradation is expected to be lower. Along with neutronic and thermal-mechanical concerns, the phase properties of the fuel and our fundamental understanding of fuel properties and fuel behavior, particularly U-Pu-Zr based fuel, is not well known.

The microstructure of irradiated U-Pu-Zr fuel exhibits three distinct concentric zones, a zirconium-enriched central zone, a zirconium-depleted and uranium-enriched intermediate zone, and a zirconium-enriched zone on the outer periphery where the effective heat of transport drives the direction of zirconium migration atoms in different phases. Phase-dependent diffusion coefficients and heats of transport are not well known, with early evidence indicating the phase transition temperatures, experimentally derived from fresh fuel, may not be consistent with irradiated fuel. Final considerations are related to the phase diagram itself. Figure 1 below 
shows the experimentally derived phase diagram for U-19Pu-10Zr fuel from Ref. (Kim et al., 2004, 2006). Noting the complexity and difficulty associated with programming logic for such a diagram, along with the lack of any material property information for several of the phases, a simplified version based on that used in (Karahan and Buongiorno, 2009) and (Kim et al., 2004, 2006) was used in practice, shown here in Figure 2.

\section{Figure 1}

\section{Figure 2}

There have been several published papers that analyze constituent distribution in metallic fuels mechanistically for U-Zr and U-Pu-Zr fuels (Karahan and Buongiorno, 2009), (Kim et al. 2004, 2006), (Hoffman et al., 1996), (Ishida et al. 1993). In the most recent paper published by (Kim et al., 2006) a new computational model was developed to solve the diffusion equations using a simplified pseudo-binary phase diagram (shown in Figure 2). The model was in one dimension (1-D) and the diffusion equation was not solved simultaneously with the thermal conduction equation due to the difficulties encountered in predicting the experimental data. In order to predict the location of the phase transitions correctly an artificial temperature gradient was imposed as a boundary condition and phasic diffusion coefficients were evaluated to match the data. However, the imposed temperature profile resulted in an unphysical heat flux that is not consistent with the operator-declared linear heating rate. The use of reported enthalpies of solution and the simplified phase diagram have inconsistencies. Karahan and Buongiorno (Karahan and Buongiorno, 2009) repeated aspects of earlier work by (Kim et al., 2004, 2006), aiming to develop an improved capability but used the same artificial temperature boundary condition in their validation of FEAST metallic fuel code (Karahan and Buongiorno, 2009). Generally speaking the validation of the metallic codes FEAST (Karahan and Buongiorno, 2009), SESAME (Kobayashi et al., 1990), ALFUS (Ogata et al., 1999), MACSIS (Hwang et al., 1998), FEMAXI-III (Nakajima et al.,1985), GERMINAL (Melis et al., 1992), TRAFFIC (Cameron et al., 1990), ALYCONE (Sercombe et al., 2009), LIFE-MATAL (Jankus et al., 1972), and BISON (Williamson et al., 2012), all suffer from similar validation deficiencies due to a sparse validation database. In addition, most of the modeling work mentioned above was in 1-D and 2-D. Despite the fact that multiple computer codes have been developed by many researchers, a robust predictive capability for quantifying fuel behavior and constituent distribution in metallic fuels and the associated uncertainty is still unavailable; large uncertainties and scatter still exist in predictions.

A new fuel performance code, BISON (Williamson et al., 2012), has been recently developed. BISON is a 3-D fuel performance analysis code developed using the MOOSE (Williamson et al., 2012) framework, a general-purpose framework for the solution of differential equations. The primary objective of this work is to use BISON to solve the coupled thermal diffusion problem with an improved formulation to reanalyze constituent redistribution in metallic fuels. Our model, as we will summarize in the next section and published previously in (Unal et al., 2013a) is similar to the model of (Kim et al., 2004, 2006) and (Ishida et al., 1993), but several significant differences are listed here. Our model corrects an inconsistency between the enthalpies of solution and the solubility limit curves of the phase diagram. It also adds an artificial diffusion term when in the 2-phase regime that stabilizes the standard Galerkin FE method used by BISON (Williamson et al., 2012). Another improvement made is in the formulation of the zirconium flux. The Soret diffusion term used in the previous metallic codes includes a zirconium 
concentration multiplied by the sum of heat of reaction and enthalpies of solution. To ensure a physical zirconium mole fraction, in the presence of a fixed plutonium mole fraction, it is necessary that the zirconium flux tend to 0 as the concentration of zirconium tends to either limiting value ( 0 or 1 less the plutonium mole fraction). This is true for the lower limit $(0)$, but not the upper limit (1). To address this issue we introduced an additional factor into the Soret term coefficient. With these new modifications we first reanalyze the data taken from rod T179 (Kim et al., 2004, 2006) of EBR-II to revaluate the previously recommended diffusion coefficients. This is important in two aspects. First, we have an experimental program measuring diffusion coefficients from diffusion-coupled experiments. The predictions reported in this paper may be validated with the diffusion-coupled data to provide a better predictive capability. Secondly, the use of a better-validated coupled solution will provide greater confidence in the design of advanced fuels. Therefore, we believe the formulation and framework presented in this paper are credible and represent a significant improvement relative to previous work.

Section 2 discusses the summary of our equation formulation and its implementation in BISON. In this paper we employ an iterative validation scheme using two data sets. We calibrate our modeling parameters (Section 3) with these two sets of data whereupon the calibrated model is qualitatively assessed against another independent set of data due to the absence of quantitative data for the experiment. Section 4 discusses the validation data in more detail. Section 5 discusses the simulation and modeling enhancements made with regard to key modeling parameters. We describe the simulation and modeling process in Section 6, with the results of the simulations discussed in section 7 and we finally present our conclusions in Section 8.

\section{Constituent Redistribution Model}

In this section we present a model (Unal et al., 2013a) of constituent migration in U-Pu-Zr fuel. It is a refinement of an earlier model (Carlson, 2009) that was based on the model developed by (Hoffman, et al., 1997) for U-Zr nuclear fuel and later used in (Kim et al. 2004, 2006) in a pseudo-binary study of U-Pu-Zr fuel. Similar models are considered in (Karahan and Buongiorno, 2009) and (Ishida et al. 1993). These models adapt the original work of (Shewmon, 1958) in the approach to 2-phase regions.

\subsection{Binary constituent redistribution model}

We begin by considering a binary substitutional alloy, $\mathrm{U}-\mathrm{Zr}$ for definiteness. Let $c_{0}$ be the density of lattice sites, which for simplicity we will assume is independent of composition and temperature, and let $x_{\mathrm{Zr}}, x_{\mathrm{U}} \geq 0, x_{\mathrm{Zr}}+x_{\mathrm{U}}=1$ be the atomic fractions of zirconium and uranium. In the presence of thermal gradients, the $\mathrm{Zr}$ atom flux in a single-phase region is

$$
\mathbf{J}_{\mathrm{Zr}}=-c_{0} D\left(\nabla x_{\mathrm{Zr}}+S \nabla T\right)
$$

where $D$ is the interdiffusion coefficient of $\mathrm{Zr}$ in the phase. The term involving $\nabla T$ is the socalled Soret effect or thermodiffusion. The coefficient $S\left(x_{\mathrm{Zr}}, T\right)$ may be positive or negative. This term contributes an advective component to the $\mathrm{Zr}$ flux directed toward lower temperatures when positive and higher temperatures when negative. To ensure physical compositions, 
$0 \leq x_{\mathrm{Zr}} \leq 1$, the flux $\mathbf{J}_{\mathrm{Zr}}$ must tend to 0 as $x_{\mathrm{Zr}}$ tends to either 0 or 1 . This leads us to take as a leading order approximation,

$$
S=x_{\mathrm{Zr}}\left(1-x_{\mathrm{Zr}}\right) \frac{Q^{\star}}{R T^{2}}
$$

Where $Q^{\star}$ is the heat of transport of $\mathrm{Zr}$ in the phase and $R$ is the gas constant. In the dilute limit $x_{\mathrm{Zr}} \ll 1$, we recover the usual form $S=x_{\mathrm{Zr}} Q^{\star} / R T^{2}$ used in previous models. The additional factor $1-x_{\mathrm{Zr}}$ ensures that $x_{\mathrm{Zr}} \leq 1$. Thus in a single-phase region the $\mathrm{Zr}$ flux is

$$
\mathbf{J}_{\mathrm{Zr}}=-c_{0} D\left(\nabla x_{\mathrm{Zr}}+x_{\mathrm{Zr}}\left(1-x_{\mathrm{Zr}}\right) \frac{Q^{\star}}{R T^{2}} \nabla T\right)
$$

where $D$ and $Q^{\star}$ are coefficients associated with the phase. It is important to recognize that the usual form of $S$ proportional to $x_{\mathrm{Zr}}$ alone is not a fundamental law, but is itself merely a leading order approximation that is only valid for small $x_{\mathrm{Zr}}$. This can be seen in two ways. First, as just noted, it gives a non-zero flux when $x_{\mathrm{Zr}}=1$ that leads to unphysical solutions with $x_{\mathrm{Zr}}>1$.

Second, $S$ and its approximations should exhibit a certain symmetry. The problem could just as well be posed in terms of $x_{U}$ with the $U$ flux $\mathbf{J}_{U}$ given by an expression analogous to (2.1). On the other hand $\mathbf{J}_{\mathrm{U}}=-\mathbf{J}_{\mathrm{Zr}}$. Both should lead to the same flux, and reconciling the two leads to the symmetry. Our approximation (2.2) exhibits this symmetry, and the necessary asymptotic behavior, while the usual form does not.

Next consider the flux in a 2-phase region very near a solubility curve defined by $x_{\mathrm{Zr}}=X(T)$. For $x_{\mathrm{Zr}} \leq X(T)$ suppose the alloy is single-phase, but for $x_{\mathrm{Zr}}>X(T)$ a second $\mathrm{Zr}$-rich precipitant phase appears. Following (Shewmon, 1958) we will assume that a local equilibrium between the two phases is maintained at each point in a temperature gradient through rapid adjustment of the phase fractions via local diffusion processes. This means the composition of the major continuous phase is fixed, $x_{\mathrm{Zr}}=X(T)$ and as a consequence

$$
\nabla x_{\mathrm{Zr}}=X^{\prime}(T) \nabla T \text {. }
$$

Assuming that the flux of $\mathrm{Zr}$ (or counter flux of $U$ ) occurs only through the continuous phase, we then have

$$
\mathbf{J}_{\mathrm{zr}}=-c_{0} D\left(X^{\prime}(T)+X(T)(1-X(T)) \frac{Q^{\star}}{R T^{2}}\right) \nabla T,
$$

where $D$ and $Q^{\star}$ are the coefficients associated with the continuous phase. For simplicity we consider solubility curves $X(T)$ satisfying

$$
\frac{d X}{d T}=X(1-X) \frac{\Delta H}{R T^{2}}, \quad X\left(T_{0}\right)=X_{0},
$$

where $\Delta H, X_{0}$, and $T_{0}$ are constant model parameters. Note that this equation uniquely determines the solubility curve. With this choice (2.5) becomes 


$$
\mathbf{J}_{\mathrm{Zr}}=-c_{0} D X(T)(1-X(T)) \frac{\Delta H+Q^{\star}}{R T^{2}} \nabla T .
$$

This approach mimics (Shewmon, 1958) who took, in the dilute limit $x_{\mathrm{Zr}} \ll 1$,

$$
\frac{d X}{d T}=X \frac{\Delta H}{R T^{2}} .
$$

The models developed in (Karahan and Buongoorno, 2009) and (Kim et al, 2004, 2006) also adopted this approach, but the expressions they use for $\Delta H$ are inconsistent with their phase diagrams, and have the wrong sign in several cases. If one desires to use arbitrary solubility curves, then equation (2.5) must be used for the flux.

The mirror situation where the alloy is single phase for $x_{\mathrm{Zr}} \geq X(T)$ and two-phase for $x_{\mathrm{Zr}}<X(T)$ is precisely the same. The $\mathrm{Zr}$ flux in the two-phase region very near the solubility curve $X(T)$ is also given by (2.7) when $X(T)$ is of the form (2.6), with the coefficients $D$ and $Q^{\star}$ those associated with the continuous phase.

\subsection{Constituent migration model for $\mathrm{U}-\mathrm{Pu}-\mathrm{Zr}$}

For U-Pu-Zr fuel it has been argued (Kim et al., 2004, 2006) that a pseudo-binary treatment, in which the Pu fraction is assumed fixed, is justified both theoretically and on the basis of experimental data that show the $\mathrm{Pu}$ is largely immobile. Thus we consider a ternary $\mathrm{U}-\mathrm{Pu}-\mathrm{Zr}$ alloy where the $\mathrm{Pu}$ fraction is a fixed, spatially uniform value $x_{\mathrm{Pu}}$. As in a true binary alloy, the

remaining $\mathrm{U}$ and $\mathrm{Zr}$ constituents flow counter to each other, and if we set $\tilde{x}_{\mathrm{Zr}}=x_{\mathrm{Zr}} /\left(1-x_{\mathrm{Pu}}\right)$ we may apply the preceding binary model to the relative $\mathrm{Zr}$ atom fraction $\tilde{x}_{\mathrm{Zr}}$. Expressing the result in terms of the original ternary atom fraction $x_{\mathrm{Zr}}$ we obtain

$$
\mathbf{J}_{\mathrm{Zr}}=-c_{0} D\left(\nabla x_{\mathrm{Zr}}+\frac{x_{\mathrm{Zr}}\left(1-x_{\mathrm{Pu}}-x_{\mathrm{Zr}}\right)}{1-x_{\mathrm{Pu}}} \frac{Q^{\star}}{R T^{2}} \nabla T\right)
$$

for the $\mathrm{Zr}$ flux in a single-phase region, where the coefficients $D$ and $Q^{\star}$ are associated with the phase, and

$$
\mathbf{J}_{\mathrm{Zr}}=-c_{0} D\left(\frac{X(T)\left(1-x_{\mathrm{Pu}}-X(T)\right)}{1-x_{\mathrm{Pu}}}\right)\left(\frac{\Delta H+Q^{\star}}{R T^{2}}\right) \nabla T
$$

for the flux in a 2-phase region very near the solubility curve $x_{\mathrm{Zr}}=X(T)$, where the coefficients $D$ and $Q^{\star}$ are those associated with the continuous phase. The solubility curves are chosen to satisfy

$$
\frac{d X}{d T}=\frac{X\left(1-x_{\mathrm{Pu}}-X\right)}{1-x_{\mathrm{Pu}}} \frac{\Delta H}{R T^{2}}, \quad X\left(T_{0}\right)=X_{0},
$$

for some choice of parameters $\Delta H, X_{0}$, and $T_{0}$, which has the solution 


$$
X(T)=\left(1-x_{\mathrm{Pu}}\right)\left\{1+\frac{1-x_{\mathrm{Pu}}-X_{0}}{X_{0}} \exp \left[\frac{\Delta H}{R}\left(\frac{1}{T}-\frac{1}{T_{0}}\right)\right]\right\}^{-1} .
$$

We use the simple pseudo-binary U-19Pu-Zr phase diagram shown in Figure 2 which is based on the phase diagrams used in (Karahan and Buongiorno, 2009) and (Kim et al, 2004, 2006). There are two 2-phase regions, $\alpha+\delta$ and $\beta+\gamma$, depending on the temperature range. The nominal transition temperatures are $T_{\alpha}=595 \mathrm{C}$ and $T_{\beta}=650 \mathrm{C}$, but these two values were varied in the simulations presented in this paper. The parameters defining the solubility curves are given here in Table 1.

\section{Table 1}

The 2-phase flux (2.10) only applies very near a solubility curve and supposes that all the flux occurs through the major continuous phase, ignoring the precipitant phase. This is a reasonable assumption near a solubility limit, but we need an expression for the flux that spans the entire 2phase region from the $\mathrm{Zr}$-poor phase solubility limit to the $\mathrm{Zr}$-rich phase solubility limit. For this we use a simple weighted average of the two fluxes that apply near the solubility limits. For the $\beta+\gamma$ region we take

$$
\begin{aligned}
\mathbf{J}_{\mathrm{Zr}}=-w & c_{0} D_{\beta}\left(\frac{X_{\beta}(T)\left(1-x_{\mathrm{Pu}}-X_{\beta}(T)\right)}{1-x_{\mathrm{Pu}}}\right)\left(\frac{\Delta H_{\beta}+Q_{\beta}^{*}}{R T^{2}}\right) \nabla T \\
& -(1-w) c_{0} D_{\gamma}\left(\frac{X_{\gamma}(T)\left(1-x_{\mathrm{Pu}}-X_{\gamma}(T)\right)}{1-x_{\mathrm{Pu}}}\right)\left(\frac{\Delta H_{\gamma}+Q_{\gamma}^{*}}{R T^{2}}\right) \nabla T .
\end{aligned}
$$

The weight factor $w$ is set equal to the $\beta$ phase fraction $f=\left(X_{\gamma}(T)-x_{\mathrm{Zr}}\right) /\left(X_{\gamma}(T)-X_{\beta}(T)\right)$. The flux in the $\alpha+\delta$ region is defined analogously. Some computational experiments were done using the more sophisticated weighting $w=e^{a} f^{b} /\left(e^{a} f^{b}+(1-f)^{b}\right)$ for parameters $a$ and $b>0$, however in the end we opted for the simple linear weighting $(a=0, b=1)$.

\subsection{BISON Implementation}

The preceding pseudo-binary model for $\mathrm{U}-19 \mathrm{Pu}-\mathrm{Zr}$ fuel was implemented in the BISON fuel performance code (Williamson et al., 2012) using a standard Galerkin finite element method. Two essential numerical modifications were made in the implementation: addition of an artificial diffusion term in the 2-phase region to stabilize the algorithm, and smoothing of the model coefficients near phase diagram curves. The full model with these modifications is described in this section.

We solve the coupled system of equations

$$
\begin{gathered}
\rho c_{p} \frac{\partial T}{\partial t}=\nabla \cdot k(x, T) \nabla T+q(\mathbf{r}, x) \\
\frac{\partial x}{\partial t}=\nabla \cdot \mathcal{D}(x, T) \nabla x+\nabla \cdot \mathcal{S}(x, T) \nabla T
\end{gathered}
$$


for an evolving temperature distribution $T(\mathbf{r}, t)$ and $\mathrm{Zr}$ atom fraction distribution $x_{\mathrm{Zr}}=x(\mathbf{r}, t)$. This is a departure from the model considered in (Kim et al., 2004, 2006) which used a fixed temperature profile. We use a variable heat source $q$ that will depend on the axial position in the fuel rod and the local $\mathrm{Zr}$ fraction (actually the local fraction of actinides, which is the complement of the $\mathrm{Zr}$ fraction). Additionally a thermal conductivity model that accounts for constituent migration as well as porosity effects was used.

In a single-phase region of the phase diagram (here $\pi$ denotes the arbitrary phase) we have

$$
\begin{gathered}
\mathcal{D}(x, T)=D_{\pi}(x, T), \\
\mathcal{S}(x, T)=D_{\pi}(x, T)\left(\frac{x\left(1-x_{\mathrm{Pu}}-x\right)}{1-x_{\mathrm{Pu}}}\right) \frac{Q_{\pi}^{\star}}{R T^{2}},
\end{gathered}
$$

where $D_{\pi}$ is the diffusivity and $Q_{\pi}^{\star}$ the heat of transport of $\mathrm{Zr}$ in the phase $\pi$.

In the 2-phase $\beta+\gamma$ region $X_{\beta}(T)<x<X_{\gamma}(T)$ bounded by the solubility limit curves $X_{\beta}$ and $X_{\gamma}$, the Soret term coefficient is

$$
\begin{aligned}
\mathcal{S}(x, T)= & f_{\beta} D_{\beta}\left(X_{\beta}(T), T\right)\left(\frac{X_{\beta}(T)\left(1-x_{\mathrm{Pu}}-X_{\beta}(T)\right)}{1-x_{\mathrm{Pu}}}\right)\left(\frac{\Delta H_{\beta}+Q_{\beta}^{*}}{R T^{2}}\right) \\
& +\left(1-f_{\beta}\right) D_{\gamma}\left(X_{\gamma}(T), T\right)\left(\frac{X_{\gamma}(T)\left(1-x_{\mathrm{Pu}}-X_{\gamma}(T)\right)}{1-x_{\mathrm{Pu}}}\right)\left(\frac{\Delta H_{\gamma}+Q_{\gamma}^{*}}{R T^{2}}\right),
\end{aligned}
$$

where $f_{\beta}=\left(X_{\gamma}(T)-x\right) /\left(X_{\gamma}(T)-X_{\beta}(T)\right)$ is the phase fraction of $\beta$ according to the lever rule. In a 2-phase region one ought to take $\mathcal{D}=0$, however this would result in equation (2.15) being purely advective with a transport velocity proportional to the temperature gradient. Mathematically this leads to jump discontinuities in the $\mathrm{Zr}$ atom fraction profile at domain boundaries and boundaries between single and 2-phase regions. Moreover, it is well-known that standard centered finite difference or Galerkin finite element schemes are unstable for pure advection, leading to spurious oscillations. Thus to stabilize our Galerkin finite element implementation we add some artificial diffusion and take

$$
\mathcal{D}(x, T)=f_{\beta} D_{\beta}\left(X_{\beta}(T), T\right) p_{\beta}+\left(1-f_{\beta}\right) D_{\gamma}\left(X_{\gamma}(T), T\right) p_{\gamma},
$$

where $p_{\beta}$ and $p_{\gamma}$ are dimensionless numerical parameters taken as small as possible while maintaining stability. In future work we plan to replace this simple stabilization approach with the SUPG (streamline upwind Petrov-Galerkin) finite element method, which can be also implemented within BISON.

The $\mathcal{D}$ and $\mathcal{S}$ coefficients in the 2-phase $\alpha+\delta$ region are defined in the analogous way.

\subsubsection{Smoothing $\mathcal{D}$ and $\mathcal{S}$}

As defined above, $\mathcal{D}$ and $\mathcal{S}$ do not vary smoothly (or even continuously) with $x$ and $T$ when crossing phase diagram boundaries, and consequently the discretized system will be much more 
difficult to solve than it would otherwise be. We have ameliorated this effect by smoothing $\mathcal{D}$ and $\mathcal{S}$ in a small $\varepsilon$-neighborhood of the phase diagram curves.

First consider the temperature interval corresponding to the 2 -phase $\beta+\gamma$ region. In the strip $\left[X_{\beta}(T), X_{\beta}(T)+\epsilon_{x}\right]$ bordering the solubility curve $X_{\beta}$ we take

$$
\mathcal{D}(x, T)=g(s) \mathcal{D}_{\beta+\gamma}(x, T)+(1-g(s)) \mathcal{D}_{\beta}(x, T), \quad s \equiv\left(x-X_{\beta}(T)\right) / \epsilon_{x},
$$

where $\mathcal{D}_{\beta}$ and $\mathcal{D}_{\beta+\gamma}$ denote the original diffusivities in the single and 2-phase regions defined above, and $g$ is the smooth function $g(s)=s^{2}(3-2 s)$. Similarly, in the strip $\left[X_{\gamma}(T)-\epsilon_{x}, X_{\gamma}(T)\right]$ bordering the solubility curve $X_{\gamma}$ we take

$$
\mathcal{D}(x, T)=g(s) \mathcal{D}_{\beta+\gamma}(x, T)+(1-g(s)) \mathcal{D}_{\gamma}(x, T), \quad s \equiv\left(X_{\gamma}(T)-x\right) / \epsilon_{x} .
$$

Otherwise we do not modify $\mathcal{D}$. The Soret coefficient $\mathcal{S}$ is smoothed in exactly the same way. Smoothing in the temperature interval corresponding to the 2-phase $\alpha+\delta$ region is done analogously.

Finally, when $\left|T-T_{0}\right|<\epsilon_{T}$, where the line $T=T_{0}$ separates two temperature slabs of the phase diagram (e.g., $\alpha-\delta$ and $\beta-\gamma$, or $\beta-\gamma$ and $\gamma$ ), we take

$$
\mathcal{D}(x, T)=(1-h(s)) \mathcal{D}_{1}(x, T)+h(s) \mathcal{D}_{2}(x, T), \quad s \equiv\left(T-T_{0}\right) / \epsilon_{T},
$$

where $\mathcal{D}_{1}$ and $\mathcal{D}_{2}$ denote the smoothed diffusion coefficients computed in the temperature slab below and above $T=T_{0}$ as just described, and $h$ is the smooth function $h(s)=\frac{1}{4}(2-s)(s+1)^{2}$. Otherwise we do not modify the smoothed diffusion coefficient of a time slab. Again, $\mathcal{S}$ is smoothed in exactly the same way.

\subsection{Modeling Parameters}

There are many inputs needed for the fuel performance code BISON and all are not listed here. However a subset of inputs was determined to be either important for the simulations and worth mentioning, or newly added inputs for the constituent redistribution kernel and are given here.

- System power

- Linear power for a specific height for single level 2-D simulations

- $\quad$ Rod average linear power scaled with axial power profile for full length 2D-RZ simulations

- Phase transition temperatures

- Alpha-delta to beta-gamma transition temperature quoted to be $595{ }^{\circ} \mathrm{C}$

- Beta-gamma to gamma transition temperature $650{ }^{\circ} \mathrm{C}$

- Rod edge convective and axial t-infinity boundary condition for full length $2 \mathrm{D}-\mathrm{RZ}$ simulations

- Fuel surface temperature boundary condition for 2-D simulations

- Thermal conductivity and associated porosity modifiers 
- Phase-dependent (alpha, delta, beta and gamma) diffusion coefficients (D's) and heats of transport, with values and units given in Table 2, based on values from (Kim et al, 2004, 2006) and (Ishida et al. 1993)

\section{Table 2}

A sensitivity study was performed on these modeling parameters, with the exception of thermal conductivity and heats of transport, to assess the importance of each parameter. It was found that the parameters that control the radial location of phase transformation, those that directly affect temperature (system power, phase transition temperatures and the temperature boundary conditions) have the most significant effect, whereas parameters that deal with the rate of migration (diffusion coefficients) have a much less significant impact on the location of phase transitions. Not all cases are shown here, however two representative cases are shown that illustrate this impact. In the two cases shown, Figure 3 and Figure 4, the alpha phase diffusion coefficient and power, respectively, both were adjusted by ${ }^{+} / 20 \%$ in order to see the impact on the simulation. Clearly observed is a minimal impact by the diffusion coefficient adjustment but a significant impact in the power adjustment, greatly adjusting the Zirconium profile shape depending on higher or lower powers. The same trends as Figure 3 were observed for all phase diffusion coefficients, while the same trends as Figure 4 were observed for transition temperatures adjustments and boundary condition adjustments.

\section{Figure 3}

\section{Figure 4}

\subsection{Validation Data}

In order to assess how accurately the constituent redistribution model in BISON was predicting actual test data, three different validation cases were obtained - these cases are: fuel rod T179, Figure 5 from Ref. (Kim et al., 2004, 2006), fuel rod DP16, Figure 6 from (D. Porter, personal communication, May 1, 2013) and fuel rod T459, Figure 7 from (D. Porter, personal communication, May 1, 2013), all irradiated in EBR-II. Both T179 and DP16 have electron microprobe (EMP) measurements taken at one axial location following irradiation, providing quantitative data across the fuel rod to compare against. Fuel rod T459 did not have EMP data; however it had cross-section pictures of the rod at 5 different axial locations, providing a qualitative way to assess the accuracy along the axial length of this experiment, particularly regarding the radial location of the phase transitions.

\section{Figure 5}

\section{Figure 6}

\section{Figure 7}


The common parameters across all three sets of experimental data are given here, while the rest of the parameters particular to each set of data are given Table 3.

- EBR-II U-19Pu-10Zr (wt\%) fuel

- $\quad 371$ C EBR-II inlet coolant temperature (used in full length 2D-RZ simulations)

- Axial power profile obtained from operator data

The power history for rod T179 and T459 were not highly variable due to the shorter in-core lifetime, thus the time average linear power was assumed for the full 92 and 142 day burns. However the power history for DP16 was more variable, changing up to $10 \%$ from initial beginning of cycle power, varying higher and lower over the life of the fuel rod; thus for DP16 the power history was followed according to the operator declared data with all power changes accounted for.

\section{Table 3}

\subsection{Simulation and Modeling Enhancements}

Fuel rod T179 was the initial dataset obtained for validation of the redistribution model. This exercise immediately pointed to the need for a thermal conductivity model able to accurately capture the effects of zirconium migration on the thermal conductivity, over a wide range of zirconium concentrations. Using experimentally derived relations for $\mathrm{U}-\mathrm{Pu}-\mathrm{Zr}$ based fuels, a thermal conductivity model was implemented that best matched the fresh fuel data available for $\mathrm{U}-\mathrm{Pu}-\mathrm{Zr}$ fuels. Figure 8 shows a sample calculation of thermal conductivity at beginning of cycle (BOC) and end of cycle (EOC). The " $\mathrm{x}$ " marker, light colored line with a smooth variation across the rod radius represents thermal conductivity when the fuel is homogeneously, evenly distributed at $\mathrm{U}-19 \mathrm{Pu}-10 \mathrm{Zr}$ weight fractions. The slight drop in thermal conductivity from rod center to rod edge is due to thermal conductivity dependence on temperature. As the reactor operates and the zirconium migrates, the effect on thermal conductivity is clearly observed. Where zirconium concentration goes high (rod center) and uranium is supplanted with zirconium, the conductivity drops sharply. However where the zirconium has evacuated and uranium concentration is high, the center of the rod, the thermal conductivity is much higher since the fuel is almost solely $\mathrm{U}-19 \mathrm{Pu}$ fuel at this location. The fresh fuel conductivity model used in this paper is given in Appendix 1.

While this first model for thermal conductivity captured the effects of zirconium redistribution accurately there was no accounting for porosity degradations in the fuel. To date no robust mechanistic approaches for modeling thermal conductivity degradation due to porosity, in the presence of liquid sodium in the fuel-clad gap (causing some recovery of conductivity with sodium ingress) are known. Based upon a literature survey coupled with optical radiography pictures of the fuel, particularly those from fuel rod T459, seen in Figure 7, a modification to the thermal conductivity model was employed. This model specifies a modifying coefficient "k_coeff" multiplied by the evaluated value of thermal conductivity, a fresh thermal conductivity evaluation. This " $k$ _coeff" is not applied uniformly over the fuel however, as a phasedependence is incorporated. In the alpha, delta and alpha-delta phases (those closest to rod edge), 
the conductivity modifier is calculated to be $\left(\mathrm{k} \_\right.$coeff +1$) / 2$, this attempts to capture the porosity effects which are clearly observed on the radiographic pictures, but also allow for some recovery due to sodium infiltration. In the beta and beta-gamma phases the modifier is set to be unity, signifying no reduction in conductivity as a result of porosity. This is reflected in the images by inspecting the mid-center band (corresponding to beta, beta-gamma phases) which appears to have significantly reduced porosity compared to rod edge or rod center values. Finally in the gamma phase the modifier is set to the value of "k_coeff". Thus, for example if the user inputs a $\mathrm{k} \_$coeff value of 0.7 , evaluated conductivity would be as follows:

- Alpha, Delta, Alpha-Delta regions: $\mathrm{k}=0.85 *$ fresh_conductivity

- Beta, Beta-Gamma regions: $\mathrm{k}=$ fresh_conductivity

- Gamma region: $\mathrm{k}=0.7 *$ fresh_conductivity

This approach does not have a strong mechanistic backing; however it is based on qualitative observations of the fuel constitution and relies on porosity degradation estimates from previous work (Nam et al. 1998), and (Bauer et al. 1995), and applied consistently, and with the same coefficient, to all experimental simulations.

\section{Figure 8}

\section{Figure 9}

Once this thermal conductivity model was implemented a comparison against previous work was performed. (Kim et al, 2006) previously had simulated rod T179. In the absence of a thermal solution, a fixed temperature profile was chosen that correlated phase transition locations observed in the EMP results for fuel rod T179 with the measured temperature transition values for fresh $\mathrm{U}-\mathrm{Pu}-\mathrm{Zr}$ fuel. Where the phase change was observed, the temperature at this location was chosen to be that from unirradiated experimental data, with smoothly varying dependence in between phase transition locations. Two simulations were performed using the BISON diffusion kernel implementation, one simulation where the same temperature profile was fixed according to the temperature profile derived by (Kim, et al., 2006), as well as a second simulation that coupled to the newly implemented thermal solver with a k_coeff of unity (assuming fresh conductivity throughout the simulation). Figure 9 shows the result of both simulations. "Fixed $\mathrm{T}$ " represents the simulation with a fixed temperature profile, while "Solved T" uses the new thermal conductivity model. Additionally, in both cases the recommended single phase values for diffusion coefficients from (Kim et al., 2006) were used. The fixed temperature distribution simulation showed decent results in matching the T179 experimental data (the red, dashed results) for the zirconium profile; however the premature rise towards rod center is undesirable. A much bigger issue however is the difference between the temperature distribution guessed at and the solved temperature distribution. The difference between the two is quite significant, requiring a thermal conductivity much higher than even fresh values to match the fixed temperature distribution. It is important to remember that this simulation still used a fresh evaluation for thermal conductivity, which will undoubtedly be worse in reality with porosity degradation. The fixed temperature distribution, while a valid place to start in computing constituent redistribution, does not represent physical reality. 
A second area where updated modeling capabilities are needed surrounds the changing uranium distribution. While the initial power profile across the rod is flat, due to the homogenous isotopic distribution, as the zirconium and uranium migrate the power profile will adjust as a function of the uranium migration. To capture this effect an MCNP (MCNP, 2008) pin cell model of the fuel pin was created. Ten rings were created for the isotopics, using the experimental data over ten representative data points. Two different geometries were investigated, one with the EBR-II quoted pin pitch and a second case with a significantly larger pin pitch (thus much more sodium coolant) to assess the sensitivity to the neutron mean free path. Figure 10 shows the MCNP (MCNP, 2008) model used in both of these simulations.

\section{Figure 10}

The result of these simulations showed a swing of power of $80 \%-120 \%$ relative to the average power across the fuel rod. Additionally the investigation of mean free path effects showed essentially no sensitivity between the two simulations, indicating that the pin pitch would need to be significantly larger than a $3.0 \mathrm{~cm}$ pitch to have an effect. Thus a single fit of power fraction as a function of zirconium concentration was determined, combining the results of both simulations, as shown in Figure 11. The "Avg" data shows the 10 data points chosen to represent the 10 rings in the MCNP simulation. The $0.8 \mathrm{~cm}$ and $3.0 \mathrm{~cm}$ results show the two different simulations and how similar the results are with two very different pin pitches. The data from both the $0.8 \mathrm{~cm}$ and $3.0 \mathrm{~cm}$ simulations was fitted as a function of zirconium concentration to produce Figure 12, showing an excellent correlation between the power and the zirconium concentration.

\section{Figure 11}

\section{Figure 12}

Using the diffusion kernel enhancements in thermal conductivity and power redistribution many simulations of pin T179 were performed - varying power, transition temperatures, fuel surface temperature and the diffusion coefficients. While iterating on these tens of thousands of simulations several things became clear. First, either the power in the rod was lower than reported, or the fuel surface temperature was lower than reported, or the transition temperatures from the phase diagram are higher than reported. This is observed in the results shown in Figure 9, the "Solved T" zirconium distribution represented by the gray line with $\mathrm{Zr}$ minimum beginning at $\sim 1.6 \mathrm{~mm}$. The phase transitions should be near 0.6 and $1.2 \mathrm{~mm}$, however they are seen to be near 1.2 and $1.75 \mathrm{~mm}$. The only way these phase transitions move towards rod center is with the temperature dropping (thus either the power dropping, or the fuel surface temperature being lower), or the phase transition temperatures increasing. At this point single level 2-D simulations of just the fuel were being performed. The results of these simulations showed the need for multiple datasets to compare against, and a validation process which could utilize all datasets in a technically defensible and robust manner.

\subsection{Simulation and Modeling Process}

The simulation and modeling process used to determine optimum simulation parameters is shown below in Figure 13. The first step was largely performed in the numerous aforementioned 
single level 2-D simulations of T179. Moving from those simulations, a full length 2D-RZ model of T179 with a convective heat transfer boundary condition along the length of the rod, a known inlet coolant temperature, and conduction across the sodium gap and D9 cladding was performed. The objective was to eliminate the fuel surface temperature variable and re-optimize the diffusion coefficients. These parameters were then applied to the DP16 simulation to assess their accuracy. Following this step the parameters were optimized for DP16, then fed back into the T179 simulation, which were then again refined and fed back into the DP16 simulation as many time as needed to obtain a good solution in both cases with common parameters, diffusion coefficients and phase transition temperatures. Finally these parameters were then applied to rod T459 as an independent assessment that was not part of the iterative scheme. The comparison against T459 is qualitative since no EMP scans exist for the rod, however the radial locations of phase transitions can be estimated from the pictures with the known rod diameter, which is what was performed.

In the listing of modeling parameters above, the phase-dependent heats of transport were mentioned and listed. Recalling that the gamma phase heat of transport contains a negative sign is very important for simulation considerations. If the gamma phase heat of transport was positive just like the other phases, then generally the zirconium would all drive toward cooler conditions, toward the rod edge. However since it is negative, this means any part of the problem domain that encompasses the gamma phase will have some driving force towards rod center, illustrated in Figure 14.

\section{Figure 13}

\section{Figure 14}

\subsection{Simulation and Modeling Results - T179}

The first iteration of 2-D T179 simulations included the varying of power, fuel surface temperature, temperature transitions and diffusion coefficients. As a result of these simulations three different cases were found that matched the experimental data well, shown in Table 4 .

\section{Table 4}

Case 1 holds the transition temperatures at the literature quoted values of 595 and $650{ }^{\circ} \mathrm{C}$, allowing the power and fuel surface temperature to be changed from recommended conditions, along with varying the diffusion coefficients. Case 2 does the opposite, fixing the power and fuel surface temperature at recommended values $\left(410 \mathrm{~W} / \mathrm{cm}\right.$ and $\left.545^{\circ} \mathrm{C}\right)$ while adjusting the transition temperatures and diffusion coefficients until the data is matched well. Finally case 3 represents some average of case 1 and case 2, varying in smaller amounts the power, fuel surface temperature, transition temperatures and diffusion coefficients. It was determined that there is no unique solution to the problem if the transition temperatures, power and fuel surface temperature are treated as free variables. One obvious trend relates to the diffusion coefficient multipliers required for a good solution. In case 1, the lowest temperature solution, the enhancements to the diffusion coefficient were very high due to the colder temperature profile, however as the temperature distribution is increased to an intermediate value in case 3 , then the 
highest temperatures in case 2 , the diffusion coefficient multipliers correspondingly decreased. Hence trading off higher transition temperatures in lieu of lower power or fuel surface temperatures allows for a reduction in the diffusion coefficient enhancement required to get an accurate solution. Figure 15 shows the results for case 1, case 2 and case 3 respectively.

\section{Figure 15}

Additionally one last item study performed was the effect of thermal conductivity changes. Can the solution be accurately matched if the quoted power, fuel surface temperature, and phase transition temperatures are all used, but thermal conductivity is varied along with diffusion coefficient enhancements? Yes the solution can be matched, as Figure 16 clearly shows. However this is not a technically defensible solution. The average thermal conductivity required to match this was 2.5 times higher than fresh conductivity, where thermal conductivity is expected to degrade, not improve, with burnup. This 2.5 increase resulted in an average conductivity of $52.86 \mathrm{~W} / \mathrm{cm}^{-}{ }^{\circ} \mathrm{C}$, which is not far from pure liquid sodium values.

\section{Figure 16}

Viewing the temperature axis one can get an idea of the drastic temperature drop at fuel centerline as a result of the conductivity enhancement, where the peak centerline temperature dropped nearly $100^{\circ} \mathrm{C}$, from 695 to $605^{\circ} \mathrm{C}$. Additionally, to get the constituents to move as required in such a cold environment, the diffusion coefficient enhancements required were quite large, given in Table 5.

\section{Table 5}

Once the simulation parameters were determined from cases 1,2 and 3, neglecting the thermal conductivity study due to non-physicality, and it was noted that no unique solution was obtained with so many free variables, it was decided that a full length 2D-RZ simulation including the sodium gap and D9 cladding, coupling to a convective heat transfer calculation on the rod edge would help eliminate the fuel surface temperature variable. Using recommended correlations for the Peclet number and Nusselt number, as well as operating data retrieved for the appropriate cycles and channel of EBR-II, the convective heat transfer coefficient was calculated. Additionally, using the known inlet and outlet temperatures of the channel of interest in EBR-II, a linear profile for temperature for t-infinity (the bulk coolant temperature) was also utilized in the calculation. Figure 17 shows the results from this simulation.

\section{Figure 17}

A couple observations from the results are drawn. First, the calculated temperature at the fuel, sodium gap interface is found to be $\sim 554{ }^{\circ} \mathrm{C}$, which is fairly close to the provided value of 545 ${ }^{\circ} \mathrm{C}$. Secondly the redistributed power distribution peaking where the zirconium concentration decreases is observed in the middle plot. Clearly observed is a back diffusion that starts to occur at rod center, near the $0.6 \mathrm{z} / \mathrm{L}$ axial height of the rod, which is reproduced across the $0.67 \mathrm{z} / \mathrm{L}$ height, the axial height pertaining to T179, in case 2 results seen in Figure 18. Additionally observed is the shift in transition location slightly towards rod edge, due to the slightly hotter conditions, being $\sim 10{ }^{\circ} \mathrm{C}$ hotter than the original simulations. 
Optimizing using this full length 2D-RZ simulation resulted in phase transition temperatures of 677 and $707{ }^{\circ} \mathrm{C}$, as well as the diffusion coefficient multipliers given in Table 6, with the simulation result shown as the "Optimized Parameters" result in Figure 18.

\section{Table 6}

\section{Figure 18}

\subsection{Simulation and Modeling Results - DP16}

Once viable modeling parameters were determined from the single level 2-D and full length 2DRZ iterations on fuel rod T179, these parameters were then applied to fuel rod DP16 which is a much longer burn (485 days). The initial results for DP16 using the diffusion coefficient enhancements and transition temperatures provided in Table 6 are shown in Figure 19.

\section{Figure 19}

Clearly the results are unsatisfactory, with the back diffusion that was observed at the 92 day mark in T179 now clearing out all the zirconium from the gamma region and piling up on rod edge. This does not match the experimental distribution, except perhaps at the beta-gamma to alpha-delta transition location (near $1.6 \mathrm{~mm}$ ). Thus, this result required a re-optimization of the parameters to DP16 based values, then a reapplication to T179 and a back and forth iterative process "as needed", as shown in Figure 13. Before showing the final results of this optimization process, a quick note needs to be made about the DP16 experimental results. First notice the minimum zirconium concentration is $\sim 0.09$ mole fraction, as opposed to the $\sim 0.04$ mole fraction observed in T179, Figure 18. The 0.04 mole fraction is consistent with the fresh fuel phase diagram beta phase solubility lines, Figure 1 and Figure 2. The data will not be matched at 0.09 mole fraction without a fundamental change in the phase diagram. Additionally note the intermediate shelf between 0.6 and $0.9 \mathrm{~mm}$. Again this nuance is not captured in the simplified phase diagram used, Figure 2, nor observed in the T179 experimental data. Both of these are most likely artifacts of high burnup fuel. Once the fuel has burned to 10 at $\%$, this means that the isotopic composition of the fuel has been significantly altered. Addition of trace (1-2 at $\%)$ materials can cause noticeable changes, thus high burnup fuel is expected to behavior differently than lower burnup fuel. That said, the aim is to try to match general trends and transition locations as opposed to developing one phase diagram and one set of parameters that will exactly match all data sets. The final results of the iterative process are shown in Figure 20 for fuel rod DP16 and Figure 21 for fuel rod T179.

\section{Figure 20}

\section{Figure 21}

The final values used in both of these simulations are transition temperatures of $692^{\circ} \mathrm{C}$ and $722^{\circ} \mathrm{C}$ along with diffusion coefficient enhancement factors given in Table 7 .

\section{Table 7}

The results obtained in for the two experiments look good. In both cases operator-declared powers were used, along with best-estimate fuel surface temperature conditions. The same 
diffusion coefficient multipliers and phase transition temperatures were used. The two simulations are of drastically different lengths, 92 days and 485 days, yet the results in both cases show strong agreement with the experimental trends.

The final step in the process was to apply these parameter values to fuel rod T459 and compare the estimated phase transition locations with the simulation prediction. There are several items that need to be kept in mind. First the phase transition locations were estimated by ruler measurement on paper, scaled to the known outer diameter. Therefore there is some uncertainty associated with hand/ruler measurements on printouts of the pictures. Secondly this simulation was not utilized in the iterative optimization process, thus it represents and independent assessment of the diffusion coefficients and transition temperatures, where again the operator declared power is used along with best estimate fuel surface temperature. Three of the five results are shown here, the top, $2^{\text {nd }}$ from top and middle ( $3^{\text {rd }}$ from top) locations in Figure 22 , Figure 23 and Figure 24, respectively.

\section{Figure 22}

The results for the top location were the hardest to estimate on paper due to noticeable rod fracture during post-irradiation processing, however with the best estimate of the transition locations, shown with the dashed vertical lines, the results look decent. The simulation predicted gamma to beta transition location near $0.3 \mathrm{~mm}$ is close to the measured value, while the betagamma to alpha-delta transition temperature rose at $1.4 \mathrm{~mm}$. It is hard to make an assessment of how accurate this is since the phase transformation in the picture is not clear. It is possible that the lower power diffusion caused less clear phase distinctions, due to less zirconium movement at the lower temperature conditions. A comparison of the radiograph at this axial level versus $0.277 \mathrm{~m}$ (Figure 23) certainly supports this possibility, where the phase distinctions at $0.277 \mathrm{~m}$ are much clearer, and the beta phase (the intermediate ring) significantly better defined.

\section{Figure 23}

The results for the $2^{\text {nd }}$ from the top location, Figure 23 , were clearer with no measurable fracture and the results look very good. The simulation-predicted gamma to beta transition location near $0.5 \mathrm{~mm}$ is nearly identical to the measured value, while the beta-gamma to alpha-delta transition temperature rose at $1.05 \mathrm{~mm}$, again nearly identical to the measured value. It is interesting to note that this $0.277 \mathrm{~m}$ location correlates with a $0.80 \mathrm{z} / \mathrm{L}$ axial height, the closest location from this experiment to the 0.67 and $0.78 \mathrm{z} / \mathrm{L}$ locations of fuel rods T179 and DP16 respectively.

\section{Figure 24}

The results for the middle location, Figure 24, were also clearer with no measurable fracture, however the results look poor. The predicted gamma-to-beta transition location near $0.3 \mathrm{~mm}$ is much earlier than the measured value of $0.8 \mathrm{~mm}$, while the beta-gamma to alpha-delta transition temperature rose at $0.75 \mathrm{~mm}$, again much earlier than the measured value of $1.5 \mathrm{~mm}$. One item of interest is the less porous looking center (gamma phase) region. There potentially may be less gamma phase present and a more prevalent two-phase beta-gamma region causing a less porous center. The most stark element of the results at this height is how much further out the transition locations extend, 0.8 and $1.5 \mathrm{~mm}$, than the higher axial location shown in Figure 24, where the transition location occur at 0.5 and $1.05 \mathrm{~mm}$. A few conjectures for how this occurs: 
- The conditions at axial level $0.162 \mathrm{~m}$ are hotter than those at $0.277 \mathrm{~m}$

- The phase transition temperatures behave differently at different heights

- Phase diffusion coefficients have a different temperature dependence

It is hard to imagine option 1 being true. The axial power profile for EBR-II is quite well known with a peaking factor of 1.12 at $0.162 \mathrm{~m}$ and 0.92 at $0.277 \mathrm{~m}$, while a significant difference the coolant will also be much cooler at the lower axial level, predicted to be $30-35^{\circ} \mathrm{C}$ cooler at 0.162 $\mathrm{m}$ than $0.277 \mathrm{~m}$. This is illustrated in Figure 25 showing the temperature distribution at each height and Figure 26 showing the zirconium distribution at each height.

\section{Figure 25}

\section{Figure 26}

Seen here in Figure 25 the temperature distribution at level $0.162 \mathrm{~m}$ starts out significantly cooler than at $0.277 \mathrm{~m}$ but climbs at a rate much higher due to the higher power at this level. However by the time rod center is reached, the temperature distribution at $0.162 \mathrm{~m}$ still has not achieved equivalent status as the $0.277 \mathrm{~m}$ height, where it would need to exceed this temperature distribution by a significant margin to cause the temperature transition locations observed in the data, assuming no difference in temperature transition locations. This can also be observed in Figure 26, where the zirconium distribution at $0.162 \mathrm{~m}$ is similar to that at $0.277 \mathrm{~m}$, but has transition locations pertaining to cooler conditions.

Option 2 is intriguing, since clearly the transition locations are further out. One confounder in this is that the phase distinctions at $0.162 \mathrm{~m}$ are not nearly as clearly delineated as at $0.277 \mathrm{~m}$ and what appears to be a 2-phase slurry of beta-gamma occurring within the center region before a probable beta phase is encountered. Given the limited information in radiography which yields no concentration information and only general trends it is probably impossible to know for sure without further experimental evidence.

Option 3 is faced with the same difficulties as option 2 regarding the unknown concentrations in each phase and relatively fuzzier picture regarding phase transformations when compared to the radiography image at $0.277 \mathrm{~m}$. This option does again provide an intriguing alternative though as it does seem that there is more beta phase present in the center, reducing the gamma phase presence. While possible, this presents difficulties since diffusion coefficients generally change the rate of movement, but do not change transition locations nearly as readily. In the presence of no quantitative data and able to make only qualitative observations, without more precise data measurements no definitive conclusions can be made.

\subsection{Phase Transition Temperatures}

One of the most surprising findings was the significantly higher transition temperatures than the results of fresh fuel experiments. Experimental predictions from fresh fuel yield a transition temperature of $595^{\circ} \mathrm{C}$ for the alpha-delta to beta-gamma phase transition and $650^{\circ} \mathrm{C}$ for the betagamma to gamma transition temperature; however the values determined in the iterative process are nearly $100^{\circ} \mathrm{C}$ higher, $692^{\circ} \mathrm{C}$ and $722^{\circ} \mathrm{C}$ respectively. It is interesting to note however that it 
took nearly the same reduction in temperature $\left(90^{\circ} \mathrm{C}\right)$ through thermal conductivity tweaks, seen in Figure 16, as it took in transition temperature increase $\left(85-100^{\circ} \mathrm{C}\right)$ to achieve reasonable solutions, however the diffusion coefficient modifiers required at the higher temperatures were much lower. Studies on the properties of irradiated U-Pu-Zr fuel would be interesting to see if the raised phase transition temperatures are replicated in the laboratory setting.

\subsection{Application to AFC Fuels}

Initially this was hoped to be applied to the AFC-1 and AFC-2 fuels irradiated in the ATR, however electron microprobe scans for these fuels have not yet been performed. While the fuel here is a U-Pu-Zr based fuel and the fuel irradiated for tests AFC-1 and AFC-2 were U-Pu- Am$\mathrm{Np}-\mathrm{Zr}$ based fuels, it has been stated that (Burkes et al., 2010)

... the five components can be reduced to a three component system consisting of U-Pu$\mathrm{Zr}$ so that phase relationships can be extrapolated. Theoretically, minimal solubility exists between $U$ and Am, especially at low temperatures (Ogawa, 1993) while broad mutual solubility exists between Pu and Am (Massalski, 1990). For this reason, the Am content of the metal alloys can be summed with the Pu content. Likewise, Np has been observed to have mutual solubility in both $\mathrm{U}$ and $\mathrm{Pu}$ (Massalski, 1990) so that the $\mathrm{Np}$ content of the metal alloys can be evenly divided between the $\mathrm{U}$ and $\mathrm{Pu}$ contents.

Therefore the work here is beneficial and ready to be extended to modeling these advanced fuel tests as soon as experimental data becomes available.

\subsection{Discussion on diffusion coefficients for separate effect testing}

Table 8 summarizes our best-estimated diffusion coefficients for each phase for U-19Pu-10Zr metallic fuel. These coefficients are compared with the ones suggested by (Kim. et al., 2006)

\section{Table 8}

It is evident that the optimized diffusion coefficients do not require 2-phase enhancement factors, indicating that the new formulation and implementation in BISON offers improved modeling capabilities. However it should be noted that our multiplier for alpha phase is 15 times higher than previous work suggested and that we do use the multiplied coefficient in two-phase regions (i.e. the same formulation for the diffusion coefficient is used in the single and two phase regions).

Results indicate that the biggest uncertainty is in the gamma phase diffusion coefficient, followed by the alpha phase diffusion coefficient and thirdly the beta phase diffusion coefficient. However, results from (Kim. et al., 2006) indicate that all of diffusion coefficients starting with beta and gamma and followed by alpha and delta are equally important for uncertainty reduction. Since these results are derived from coupled solutions validated by multiple data sets, we believe our results are more credible until disproved by separate effect test data. We recommend the experimental campaign to look at gamma phase first, followed by alpha phase experiments and then beta phase diffusion coefficients. If the experimentally derived alpha or gamma phase diffusion coefficients are close to values given in Table 8, a cost savings strategy can be 
developed using this simulation capability again with separate effects diffusion coupled data eventually confirming the results.

\subsection{Summary and Conclusions}

The AFC metallic fuels currently being tested have higher zirconium and plutonium concentrations than those tested in the past in EBR reactors. Current metal fuel performance codes have limitations and deficiencies in predicting AFC fuel performance, particularly in the modeling of constituent distribution. No fully validated code exists due to sparse data and unknown modeling parameters. Our primary objective is to develop an initial analysis tool by incorporating state-of-the-art knowledge, constitutive models and properties of AFC metal fuels into the MOOSE/BISON framework in order to analyze AFC metallic fuel tests.

This paper presents a robust constituent redistribution formulation for modeling metallic nuclear fuel, with a special focus on validation for $\mathrm{U}-\mathrm{Pu}-\mathrm{Zr}$ based fuels. A robust predictive code has been successfully developed, tested and implemented in the BISON/MOOSE code framework. Experimental data from EBR-II, fuel pin T179 (Kim. et al., 2004, 2006) was used as the validation case. Using the available phase diagram, diffusion coefficients (Kim. et al., 2006) and design values of this fuel rod, a coupled thermal-diffusion problem with BISON was solved. Results with no modification to previous modeling parameters did not agree with the experimental data. This indicated that solution given in (Kim et al., 2006) relies on an unrealistic temperature profile.

Several improvements have been implemented. The first improvement considers modeling of rod power as a function of uranium distribution for fuel rod T179, calculated by MCNP (MCNP, 1998). A new fuel thermal conductivity model was developed to consider the full zirconium spectrum from 0 to 100 percent. The diffusion equation was revised to force the zirconium flux to go to zero when the concentration approaches zero and (1-Conc $\mathrm{Pu}_{\mathrm{Pu}}$ ), the upper and lower bounds. Initial sensitivity studies were performed using operational data and educated guesses for phase-dependent material properties, since $\mathrm{U}-\mathrm{Pu}-\mathrm{Zr}$ fuel has multiple phases present, where most of these properties have not been experimentally derived. The resulting zirconium distributions were compared against the experimentally measured distribution for fuel pin T179. In this sensitivity study, three cases were obtained that showed strong agreement with the experimental distribution. These cases required perturbing the rod power, edge temperature boundary condition, or transition temperatures on the phase diagram.

Obtained from the sensitivity study were a series of material properties, phase diffusion coefficients for instance, along with rod powers, temperatures and phase diagram characteristics. Moving on from a model of a single axial slice, the next case was a full rod model for fuel pin T179 with a convective heat transfer boundary condition that allowed the heat to conduct across the rod, sodium gap and D9 cladding. This model then gave insights into some of the bounding conditions, particularly regarding the fuel edge temperature boundary condition that should be used. 
Finally, a robust formulation for constituent redistribution was developed and implemented into advanced BISON code. The model validation included testing against experimental data from fuel pin T179 from EBR-II. A series of viable material properties for U-Pu-Zr based materials was determined through a sensitivity study on T179 which resulted in three cases with differing parameters that showed strong agreement with experimental data. Subsequently a full-scale model of T179 was simulated to reduce uncertainties, particularly relating to the temperature boundary condition for the fuel. Optimized diffusion coefficients suggest relative improvements in comparison to previous values reported by (Kim et al., 2004, 2006). The most influential or uncertain phase is found to be the gamma phase. Recommendations are made for the experimental campaign to focus on this phase first. We believe the model presented in this paper represents a strong improvement and can be used for scoping calculations with higher confidence, provided the fuel is not in design certification process yet. Further separate effect testing data is required to declare that the model is predictive enough to be used in fuel qualification calculations. Nevertheless we believe the MOOSE/BISON framework together with the constituent redistribution model developed in this study can be further enhanced in future work to yield a more robust predictive capability. We will recalibrate the coefficients by solving the fully coupled mechanical-thermal-diffusion equation simultaneously. Modifications to these diffusion coefficients are likely as more data become available - in this sense, the recommendations for diffusion coefficients made here should not be considered final, rather this study is an initial but significant step toward a more robust quantification of these coefficients.

\section{Acknowledgments}

We would like to thank Jason Hales, Richard Williamson, Pavel Medvedev, Derek Gaston, Jon Carmack and Richard Martineau for the help and support they provided with regards to the BISON code.

We are very grateful to Kemal Pasamehmetoglu for his vision on the development of science based advanced fuel development program and the support and encouragement he provided to develop the current analysis capability.

We also would like to thank DOE program manager Frank Goldner for his continuous support and the leadership he provides for the development of advanced fuels.

\section{Appendix 1: New Improved Thermal Conductivity Model for U-Pu-Zr Metallic Fuels}

(Karahan, 2010) tried to assess some of the AFC tests that included high zirconium concentrations and found that the thermal conductivity model he used in FEAST (Karahan and Buongiorno, 2009) needed to be modified to accommodate the higher zirconium concentration fuels due to it being a much stiffer fuel. Modifications to the fuel thermal conductivity, creep, modulus of elasticity and thermal expansion and fission gas diffusivity models were required to predict the data from the AFC-1F irradiation test.

We have performed a comprehensive sensitivity study (Unal et al., 2013b) using the LIFEMETAL code. The objective of the sensitivity study was to identify important macroscopic parameters of interest to modeling and simulation of metallic fuel performance. The sensitivity study was performed using data from 6 EBR-II pins. A sensitivity ranking methodology was 
deployed to narrow down the selected parameters for the current study. There were approximately 84 calibration parameters in the FEAST-Metal code, of which 32 were ultimately used in Phase II of our study (Unal et al., 2013b).

The fuel thermal conductivity and gas release models were most influential in terms of explaining overall output variability, and are therefore targets for additional calibration to further constrain their parameters. We considered all results to recommend a ranking of models that can be considered for further improvements. Our model ranking is as follows:

- Fission gas release and swelling,

- Fuel and clad conductivity,

- Species diffusion, and

- Fuel creep.

In this paper we solve only the thermal-diffusion problem and therefore are not modeling gas release, swelling or mechanical behavior yet as these will be done in the future research. In order to reduce the uncertainty in our result we wanted to make sure we used the best thermal conductivity model available. We considered the model suggested by (Karahan, 2010) however it was unclear if his suggested model predicted the fresh fuel thermal conductivity data, where it is distinctly possible that all the data may not have been available to him. After collecting all available data from (Kim and Hoffman), (Touloukian et al., 1970), (Takahashi et al., 1988) and (IFR, 1988) an new thermal conductivity model considering all available data points was generated.

Karahan suggested following modification.

$$
\begin{aligned}
& k_{U-Z r}=\left(1.0-\sqrt{\left(1-x_{Z r}\right)}\right) k_{Z r}+\sqrt{\left(1-x_{Z r}\right)}\left(x_{Z r} k_{c, U}+\left(1-x_{Z r}\right) k_{U}\right) \\
& k_{P u-Z r}=\left(1.0-\sqrt{\left(1-x_{P u}\right)}\right) k_{P u}+\sqrt{\left(1-x_{P u}\right)} k_{c, Z r} \\
& k_{U-P u-Z r}=x x_{U} k_{U-Z r}+x x_{P u} k_{P u-Z r} \\
& x_{x_{U}}+x x_{P u}=1
\end{aligned}
$$

This model over predicted the dataset with an average of $5 \mathrm{~W} / \mathrm{m}-\mathrm{K}$ and a standard deviation of $1.57 \mathrm{~W} / \mathrm{m}-\mathrm{K}$. We considered the formulation given (Touloukian et al., 1970) and adjusted the coefficient to minimize the standard deviation of error. The following equation set for evaluation of the thermal conductivity of $\mathrm{U}-\mathrm{Pu}-\mathrm{Zr}$ metallic fresh fuel is obtained.

$$
k_{U-P u-Z r}=\left(\frac{a}{a+b}\right)^{2} k_{U-Z r}+\left(\frac{b}{a+b}\right)^{2} k_{P u-Z r}+1.8\left(\frac{a}{a+b}\right)\left(\frac{b}{a+b}\right)\left(\frac{k_{U-Z r}+k_{P u-Z r}}{k_{U-Z r} k_{P u-Z r}}\right)
$$


where,

$$
\begin{aligned}
& k_{U-Z r}=\left(1.0-\sqrt{\left(1-x_{Z r}\right)}\right) k_{Z r}+\sqrt{\left(1-x_{Z r}\right)}\left(x_{Z r} k_{c, U}+\left(1-x_{Z r}\right) k_{U}\right) \\
& k_{P u-Z r}=\left(1.0-\sqrt{\left(1-x_{P u}\right)}\right) k_{P u}+\sqrt{\left(1-x_{P u}\right)} k_{c, Z r} \\
& k_{U}=21.73+1.591 E-2 T+5.907 E-6 T^{2} \\
& k_{Z r}=8.853+7.082 E-3 T+2.533 E-6 T^{2}+2.992 E 3 T^{-1} \\
& k_{P u}=4.301 E-5 T^{2}+0.08512 T-15.529 \\
& k_{c, U r}=-102.200 .1 x_{Z r}-109.2 x_{Z r}^{2}+9.435 E-3 T+3.459 E-5 T^{2}-0.02093 x_{Z r}^{T} \\
& k_{c, Z r}=29.469-118.8111 x_{P u}+88.893 x_{P u}^{2}+0.0117 T+1.922 E-5 T^{2}-0.00716 x_{P u}^{T}
\end{aligned}
$$

This modified model gives an average of $0.2 \mathrm{~W} / \mathrm{m}-\mathrm{K}$ and a standard deviation of $1.38 \mathrm{~W} / \mathrm{m}-\mathrm{K}$, eliminating over-prediction. The equation given for uranium and zirconium gives an average of $0.01 \mathrm{~W} / \mathrm{m}-\mathrm{K}$ and a standard deviation of $0.47 \mathrm{~W} / \mathrm{m}-\mathrm{K}$ indicating that equation predicts the data very well for zirconium concentration from 0 to $100 \%$.

In these equations temperature is in Kelvin and thermal conductivity is $\mathrm{W} / \mathrm{m}-\mathrm{K}$ given for fuel without irradiation effects. Irradiation effects are discussed in the main text. 


\section{References}

Advanced Fuels Campaign 2012 Accomplishment report, INL/EXT-12-276674, FCRDFUEL-2013-00032, November.

Bauer, T., et al., 1995. "In-Pile Measurement of the Thermal Conductivity of Irradiated Metallic Fuel”, Nuclear Technology, Vol. 110, pp. 407-421.

Burkes, D.E., et al., 2010. "Phase characteristics of a number of U-Pu-Am-Np-Zr metallic alloys for use as fast reactor fuels", Journal of Nuclear Materials, Vol. 396, pp. 49-56.

Cameron, R.F., et al., 1990. "Development and Application of the TRAFFIC Code for the Analysis of Fast-Reactor Safety Experiments", Nucl Energ-J BR Nucl, 29 (2) pp. 141147.

Carmack, W.J., et al., 2009. "Metallic fuels for advanced reactors", Journal of Nuclear Materials, Vol. 293, Issue 2, pp.176-183, 15 July.

Carlson, N.N., 2009, Final Report for Work Package LA0915090116: Fuel Performance Simulations, LA-UR-09-07340.

Hofman, G.L., et al, 1997. "Metallic Fast Reactor Fuels," Progress in Nuclear Energy, Vol. 31, pp. 83-110.

Hofman, G.L., et al. 1996. "Temperature gradient driven constituent redistribution in UZr alloys", Journal of Nuclear Materials, Vol. 227, Issue 3, pp.277-286.

Hwang, W., et al., 1998. "MACSIS: A Metallic Fuel Performance Analysis Code for Simulating In-Reactor Behavior under Steady-State Conditions", Nuclear Technology, Vol. 123, pp. 130-141.

Ishida, M., et al., 1993. "Constituent Migration Model for U-Pu-Zr metallic fast reactor fuel”, Nuclear Technology, Vol. 104, pp. 37.

Jankus , V.Z. and Weeks, R.W., 1972, “LIFE-II - A Computer Analysis of Fast-Reactor Fuel-Element Behavior as a Function of Reactor Operating History", Nuclear Engineering and Design, Vol. 18, pp. 83-96.

Karahan A. and Buongiorno, J., 2009. "Modeling of Thermo-Mechanical and Irradiation Behavior of Metallic and Oxide Fuels for Sodium Fast Reactors", MIT Report, MITNFC-TR-110, August.

Karahan, A., 2010. "Extending FEAST-METAL for Analysis of Actinide Bearing zirconium Rich Fuels for Sodium Fast Reactors", Nuclear Materials 2010 conference, Karlshure, Germany, October 4-7.

Kim, Y.S., et al., 2004. "Constituent redistribution in U-Pu-Zr fuel during irradiation", Journal of Nuclear Materials, Vol. 327, pp.27-36. 
Kim, Y.S., et al., 2006. "Modeling of Constituent Redistribution in U-Pu-Zr metallic Fuel, Journal of Nuclear Materials", Journal of Nuclear Materials, Vol. 359, pp.17-28.

Kim, Y.S. and Hofman, G.L., 2003: AAA Fuels Handbook, ANL-AAA-068, Argonne National Laboratory.

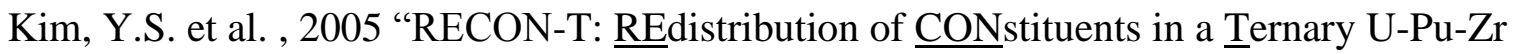
Fuel”, Trans. Am. Nucl. Soc. 93 pp. 755-756.

Kim, Y.S., et al., 2009. "Advances in Modeling of Constituent Redistribution in Fast Reactor U-Pu-Zr Fuel”, Proceedings of GLOBAL 09, Paper 9648, Paris, France, September 6-11.

Kobayashi, T., et al., 1990. "Development of the SESAME Metallic Fuel Performance Code”, Nuclear Technology, Vol. 89, pp. 183-193.

Marino, G.P., 1972. "A Numerical Calculation of the Redistribution of an Interstitial Solute in a Thermal Gradient", Nuclear Science Engineering, Vol. 49, pp. 93-98.

Massalski, T.B., (Ed.), 1990. "Binary Alloy Phase Diagrams”, second ed., vol. 3, ASM International.

Melis, J.C et al., 1992, "GERMINAL - A computer code for predicting fuel pin behavior", Journal of Nuclear Materials, Vol. 188, pp. 303-307.

Monte Carlo Team, MCNP, 2008. “A General Monte Carlo N-Particle Transport Code, Version 5, Volume I: Overview and Theory”, Los Alamos National Laboratory report LA-UR-03-1987.

Nakajima, T., et al., 1985. "FEMAXI-III: A computer Code for the Analysis of Thermal and Mechanical Behavior of Fuel Rods", JAERI-1298, Japan Atomic Energy Research Institute.

Nam, C. et al., 1998. “A Calculation Model for Fuel Constituent Redistribution and Temperature Distribution on Metallic U-10Zr Fuel Slug of Liquid Metal Reactors”, Journal of the Korean Nuclear Society, Vol. 30(6): pp. 507-517.

Ogata, T., et al., 1999, "Development and Validation of ALFUS: An irradiation Behavior Analysis Code for Metallic Fast Reactor Fuels", Nuclear Technology, Vol. 128, pp. 113123

Ogawa, T., 1993. "Alloying behaviour among U, Np, Pu and Am predicted with the Brewer valence bond model" Journal of Alloys and Compounds, Vol. 194 (1), pp. 1-7.

Sercombe, J.et al., 2009. "Multi-dimensional modeling of PCMI during base irradiation and ramp testing with ALCYONE V1. 1", Proc. Top Fuel 2009, Paris, France, pp. 130131.

Shewmon, P.C., 1958. "The redistribution of a second phase during annealing in a temperature gradient”, Trans. TMS-AIME 212. 
Sohn, Y.H. et al., 2000, "Analysis of constituent redistribution in the $\gamma$ (bcc) U-Pu-Zr alloys under gradients of temperature and concentrations" Journal of Nuclear Materials, Vol. 279, pp. 317-329.

Touloukian, Y.S., et al., 1970. "Thermophysical Properties of Matter, vol. 2, Thermal Conductivity”, pp. 675, IFI/Plenum, New York-Washington.

Takahashi, Y., et al., 1988. "Thermophysical properties of uranium-zirconium alloys" Journal of Nuclear Materials, Vol. 154, pp. 141-144.

Unal, C., et al., 2013a. "Preliminary Simulation Results of the Constituent Distribution Model Implemented into the BISON Framework for the Analysis of Performance of Metallic Fuels, International Conference on Fast Reactors and Related Fuel Cycles: Safe Technologies and Sustainable Scenarios (FR13)”, March 4-7, Paris France.

Unal, C. et al., 2013b. “Application of advanced validation concepts to oxide fuel performance codes: LIFE-4 fast-reactor and FRAPCON thermal-reactor fuel performance codes”, Nuclear Engineering and Design, Volume 263, pp. 102-128, October.

Walters, L.C., 1999. "Thirty years of fuels and materials information from EBR-II, Journal of Nuclear Materials", Vol. 270, pp. 30-43.

Williamson, R.L., et al., 2012. "Multidimensional multi-physics simulation of nuclear fuel behavior". Journal of Nuclear Materials, Vol. 423(1-3): pp. 149-163. 
Table 1 - Parameters used in BISON phase diagram

\begin{tabular}{|c|c|c|c|}
\hline Phase & $X_{0}$ (solubility intersection) & $T_{0}\left({ }^{\circ} \mathrm{C}\right)($ upper transition $\mathrm{T})$ & $\Delta H(\mathrm{~kJ} / \mathrm{mol})$ \\
\hline$\alpha$ & 0.04 & 595 & 100 \\
\hline$\delta$ & 0.70 & 595 & -3 \\
\hline$\beta$ & 0.033 & 650 & -50 \\
\hline$\gamma$ & 0.43 & 650 & -100 \\
\hline
\end{tabular}

Table 2: Diffusion coefficients, $D=D 0 \exp (-Q / R T)$, and heats of transport for U-Pu-Zr

\begin{tabular}{|c|c|c|c|c|}
\hline Phase & $\alpha$ & $\delta$ & $\beta$ & $\gamma$ \\
\hline$D_{0}\left(\mathrm{~m}^{2} / \mathrm{s}\right)$ & $3.0 \times 10^{-6}$ & $3.0 \times 10^{-6}$ & $1.14 \times 10^{-5}$ & $10^{-5.1-8.05 c+9.13 c^{2}}$ \\
\hline$Q(\mathrm{~kJ} / \mathrm{mol})$ & 170 & 150 & 180 & $128-107 \mathrm{c}+174 c^{2}$ \\
\hline$Q^{\star}(\mathrm{kJ} / \mathrm{mol})$ & 200 & 160 & 450 & -200 \\
\hline
\end{tabular}

Table 3: Experimental data for fuel rods T179, DP16 and T459

\begin{tabular}{|c|c|c|c|}
\cline { 2 - 4 } \multicolumn{1}{c|}{} & T179 & DP16 & T459 \\
\hline Burnup (at\%) & 2 & 10 & 3 \\
\hline Irradiation length (days) & 92 & 485 & 142 \\
\hline z/L location & 0.67 & 0.78 & Multiple \\
\hline EMP scan & Yes & Yes & No \\
\hline Number of axial locations & 1 & 1 & 5 \\
\hline Peaking factor & 1.06 & 0.993 & N/A (5 locations) \\
\hline Fuel surface temperature $\left({ }^{\circ} \mathrm{C}\right)$ & 545 & 550 & N/A (5 locations) \\
\hline Linear power average & 410 & 450 & 375 \\
\hline Outlet coolant temperature $\left({ }^{\circ} \mathrm{C}\right)$ & 518 & 522 & 542 \\
\hline
\end{tabular}


Table 4: T179 single level 2-D modeling parameters

\begin{tabular}{|c|c|c|c|c|c|c|c|c|}
\hline & & & & & & & \\
\hline & & Starting & Case 1 & $\Delta$ & Case 2 & $\underline{\underline{\Delta}}$ & Case 3 & $\underline{\underline{\Delta}}$ \\
\hline Power & $\underline{W} / \mathrm{cm}$ & 410 & 360 & $-12 \%$ & 410 & $\mathrm{~N} / \mathrm{A}$ & 380 & $-7 \%$ \\
\hline FST & ${ }^{\circ} \mathrm{C}$ & 545 & 485 & $-11 \%$ & 545 & $0 \%$ & 520 & $-5 \%$ \\
\hline \multirow{4}{*}{ 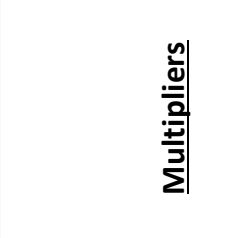 } & Alpha & 1 & 400 & $400 x$ & 100 & $100 x$ & 200 & $200 x$ \\
\hline & $\underline{\text { Delta }}$ & 1 & 5 & $5 x$ & 2 & $2 x$ & 4 & $4 x$ \\
\hline & Beta & 1 & 125 & $125 x$ & 55 & $55 x$ & 100 & $100 x$ \\
\hline & Gamma & 1 & 8 & $8 x$ & 2.5 & $2.5 x$ & 2.5 & $2.5 x$ \\
\hline A-D Transition T & ${ }^{\circ} \mathrm{C}$ & 595 & 595 & $0 \%$ & 663 & $11 \%$ & 635 & $7 \%$ \\
\hline B-G Transition T & ${ }^{\circ} \mathrm{C}$ & 650 & 650 & $0 \%$ & 695 & $7 \%$ & 661 & $2 \%$ \\
\hline
\end{tabular}

Table 5: Thermal conductivity adjusted diffusion coefficient multipliers

\begin{tabular}{|c|c|c|c|}
\hline Alpha & Delta & Beta & Gamma \\
\hline 1000 & 5 & 380 & 19 \\
\hline
\end{tabular}

Table 6: Full length 2D-RZ optimized diffusion coefficient multipliers

\begin{tabular}{|c|c|c|c|}
\hline Alpha & Delta & Beta & Gamma \\
\hline 50 & 2 & 30 & 5 \\
\hline
\end{tabular}

Table 7: Re-optimized diffusion coefficient multipliers

\begin{tabular}{|c|c|c|c|}
\hline Alpha & Delta & Beta & Gamma \\
\hline 15 & 1 & 2 & 7 \\
\hline
\end{tabular}


Table 8: Optimized Diffusion Coefficients

\begin{tabular}{|c|c|c|c|}
\hline Phase & Kim, et al. $\mathrm{D}_{\text {coeff }}$ & New $\mathrm{D}_{\text {coeff }}$ & Ratio \\
\hline Alpha & $3.00 \mathrm{E}-06$ & $15^{*} 3 \mathrm{e}-6$ & 15 \\
\hline Delta & $3.00 \mathrm{E}-06$ & $3.00 \mathrm{E}-06$ & 1 \\
\hline Beta & $1.14 \mathrm{E}-05$ & $2 * 1.14 \mathrm{e}-5$ & 2 \\
\hline Gamma & $2.6 \mathrm{E}-2$ to 0.26 & $7 \mathrm{E}-5$ to $7 \mathrm{E}-4$ & 0.003 \\
\hline A+D & $6^{*}$ (alpha+delta) & alpha+delta & 1.3333 \\
B+G & $10^{*}$ (beta+gamma) & beta+gamma & 0.0093 \\
\hline
\end{tabular}




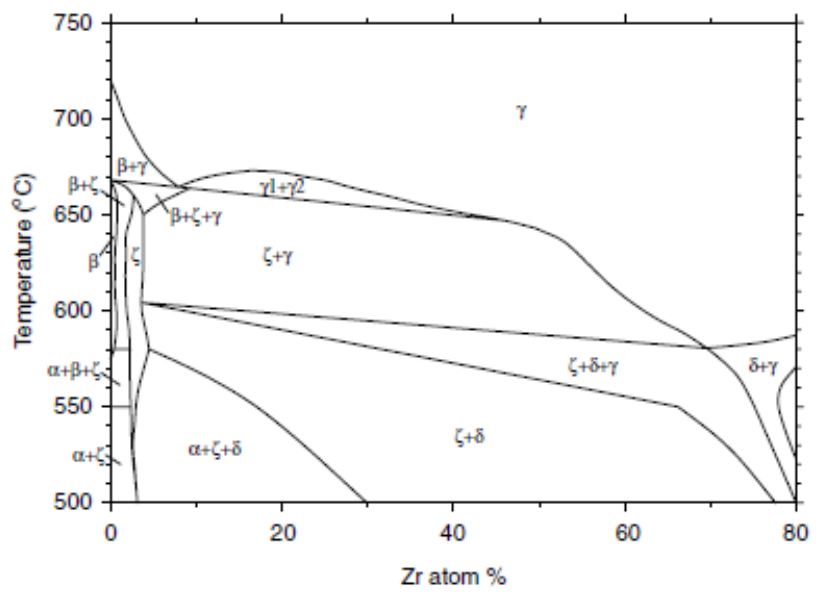

Figure 1: Pseudo-binary (U-Pu)-Zr phase diagram, Pu content fixed at 19 wt $\%$ and $10 \% \mathrm{Zr}$ from Ref (Kim et al., 2006), (Ishida et al. 1993)

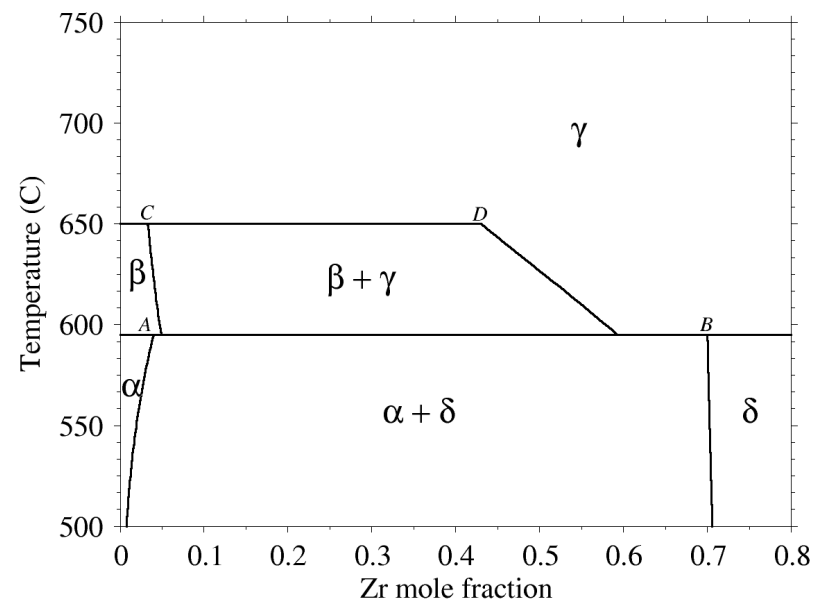

Figure 2: Pseudo-binary (U-Pu)-Zr phase diagram used in BISON model 


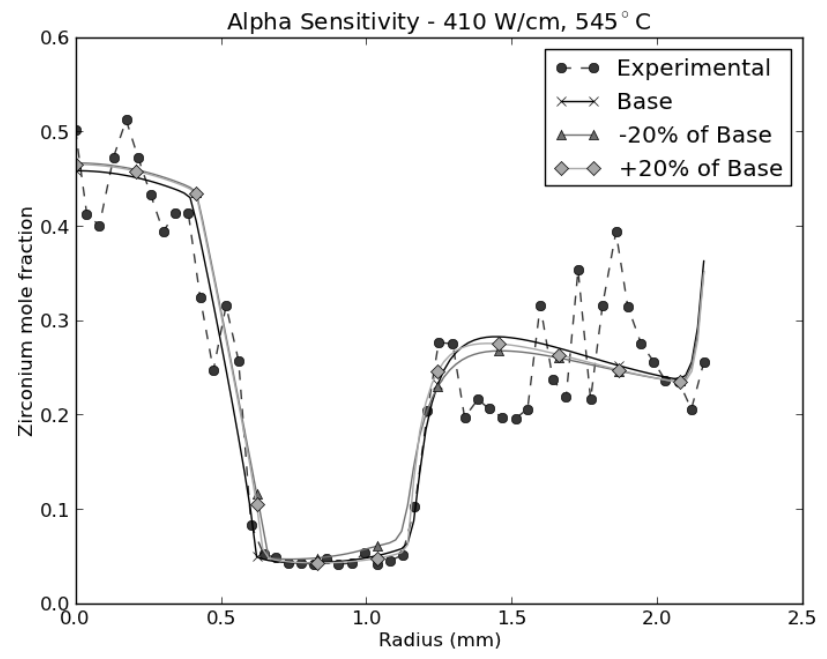

Figure 3: Zirconium distribution alpha phase diffusion coefficient sensitivity

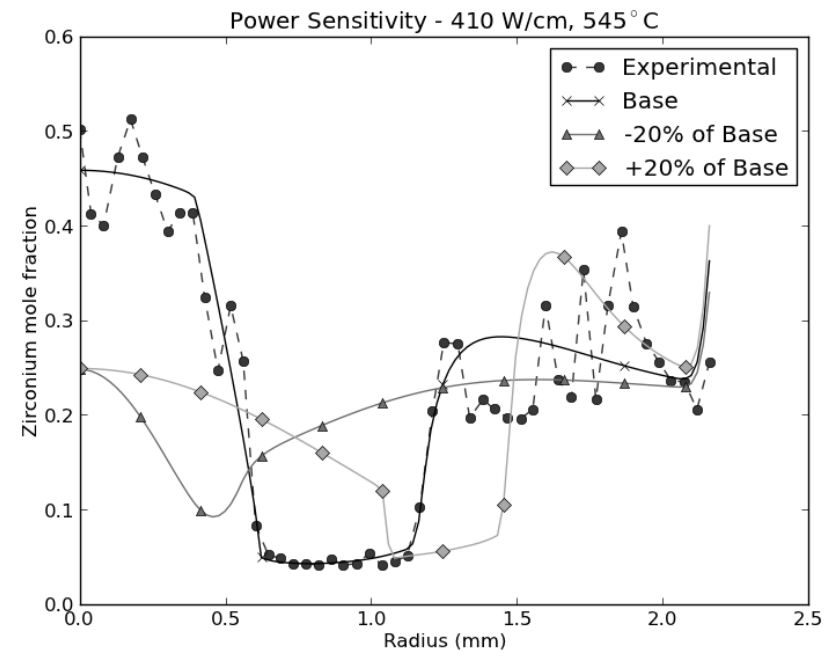

Figure 4: Zirconium distribution power sensitivity

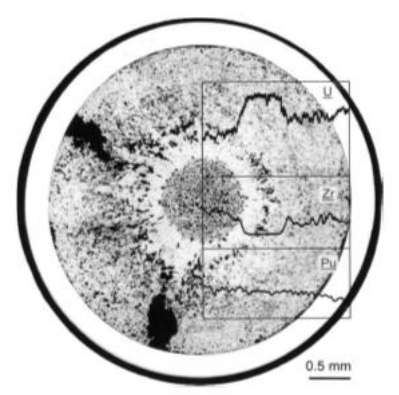

Figure 5: T179 metallographic 


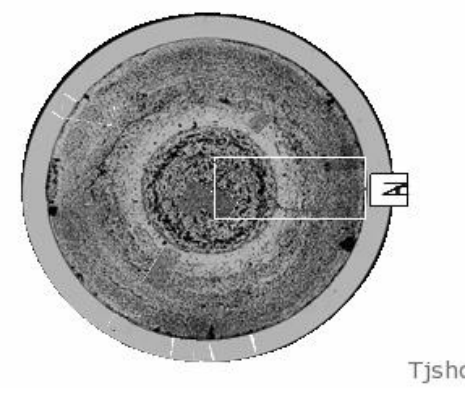

Figure 6: DP16 metallographic

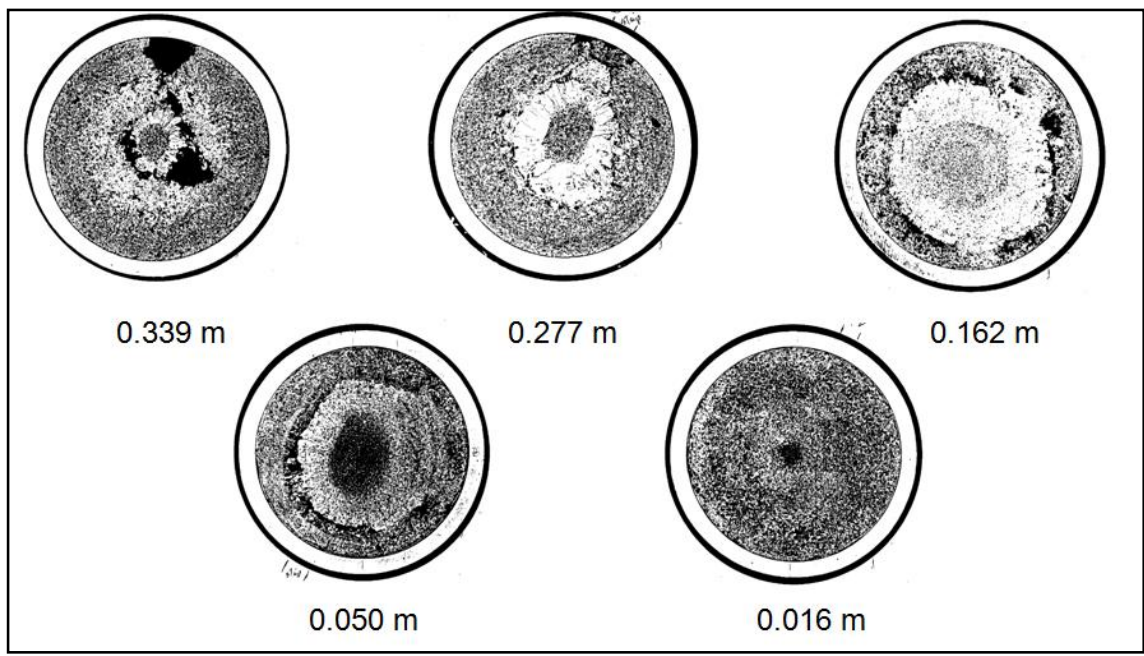

Figure 7: T459 metallographic at 5 axial heights

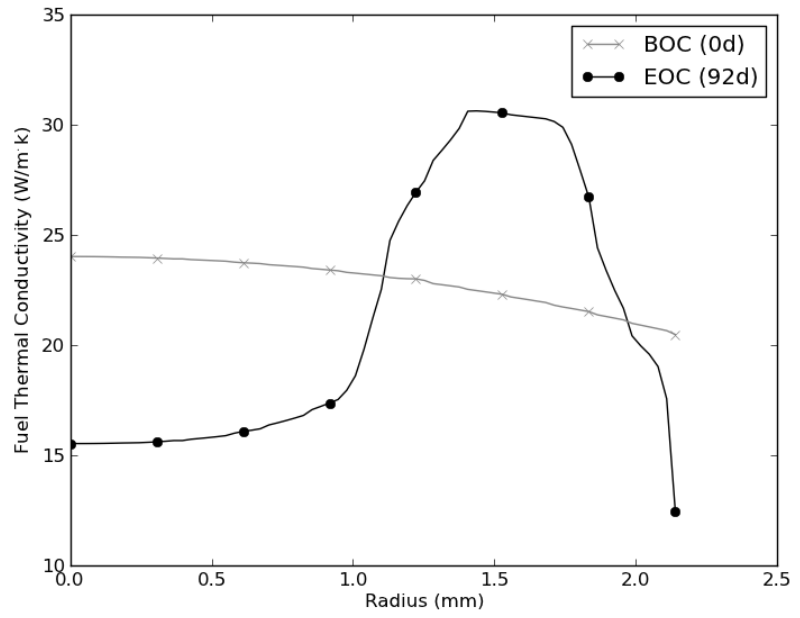

Figure 8: Thermal conductivity change along fuel radius $(\mathrm{mm})$ as a function of irradiation dependent $\mathrm{Zr}$ redistribution, beginning of life ( 0 days) and end of life (92 days) 


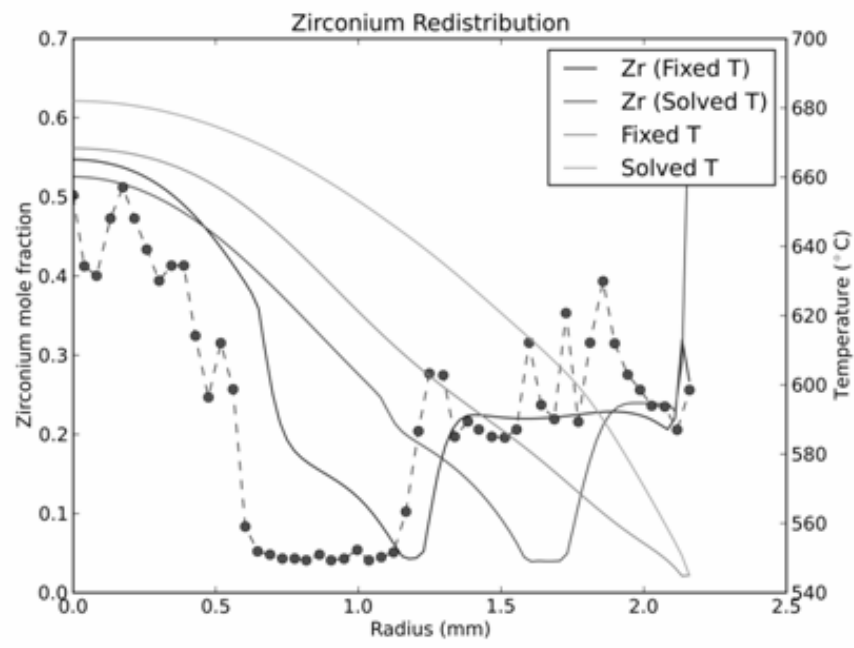

Figure 9: Comparison to previous work, experimental Zirconium distribution represented by dashed line with solid dots
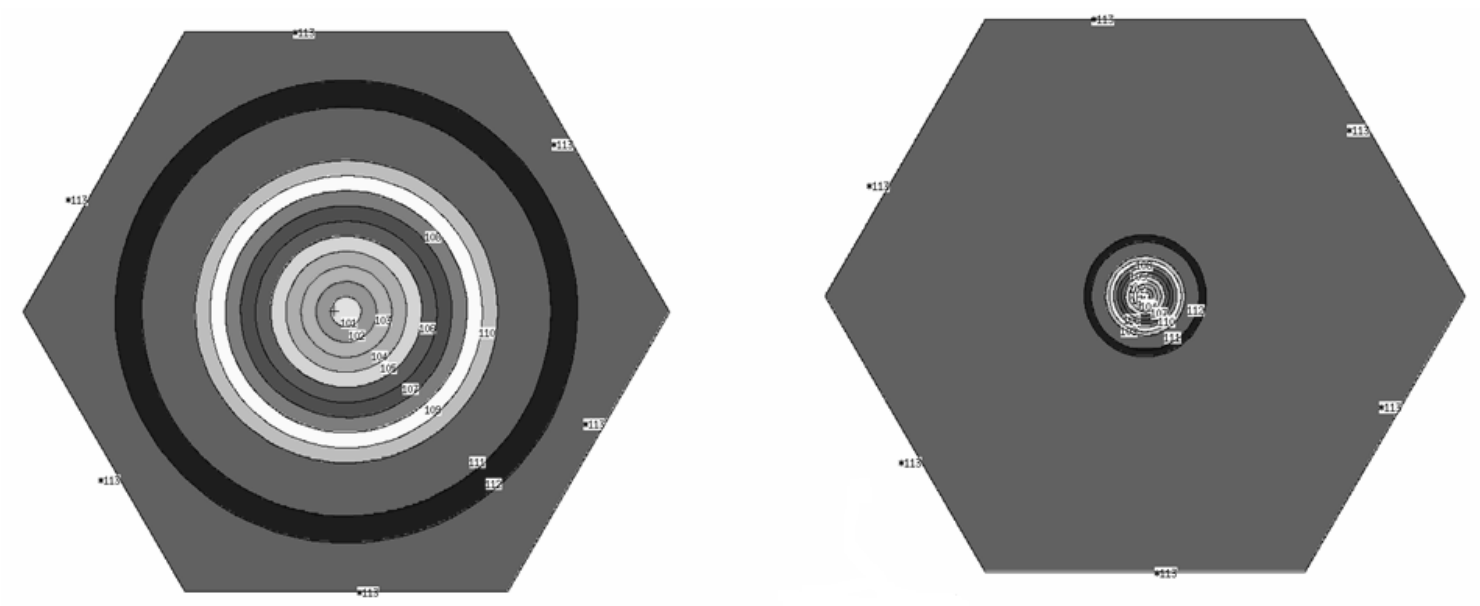

Figure 10: MCNP model for power distribution calculation, two different pin pitches investigated 


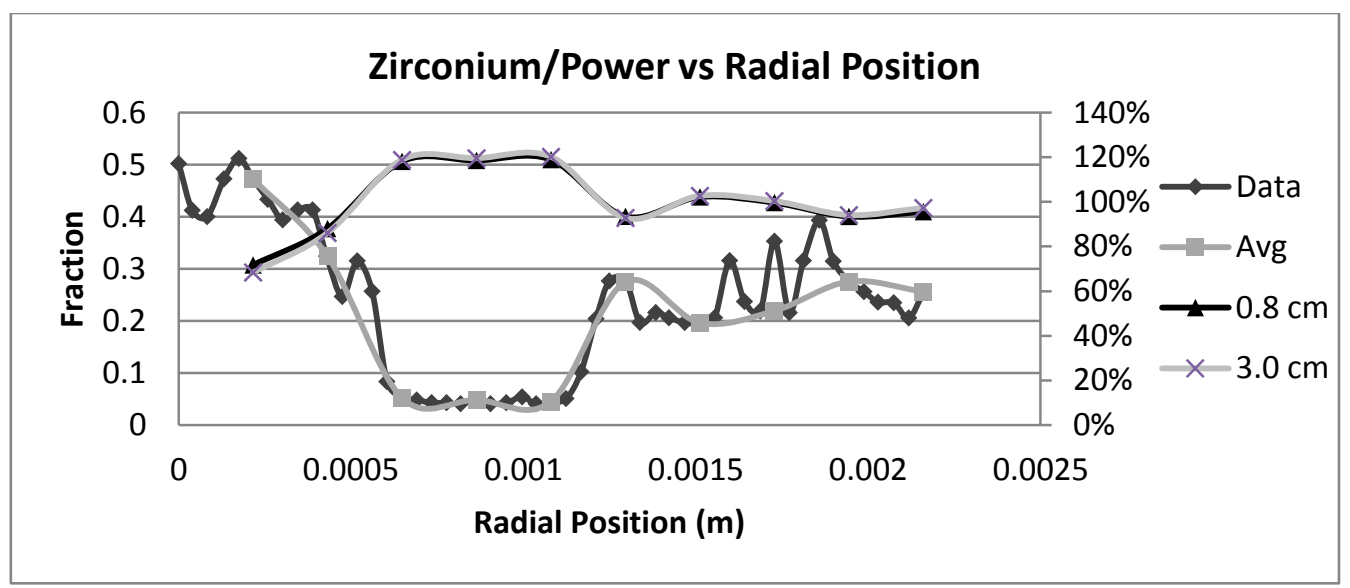

Figure 11: Zirconium and power fraction as a function of radial position, where Zr fraction is low power is correspondingly high

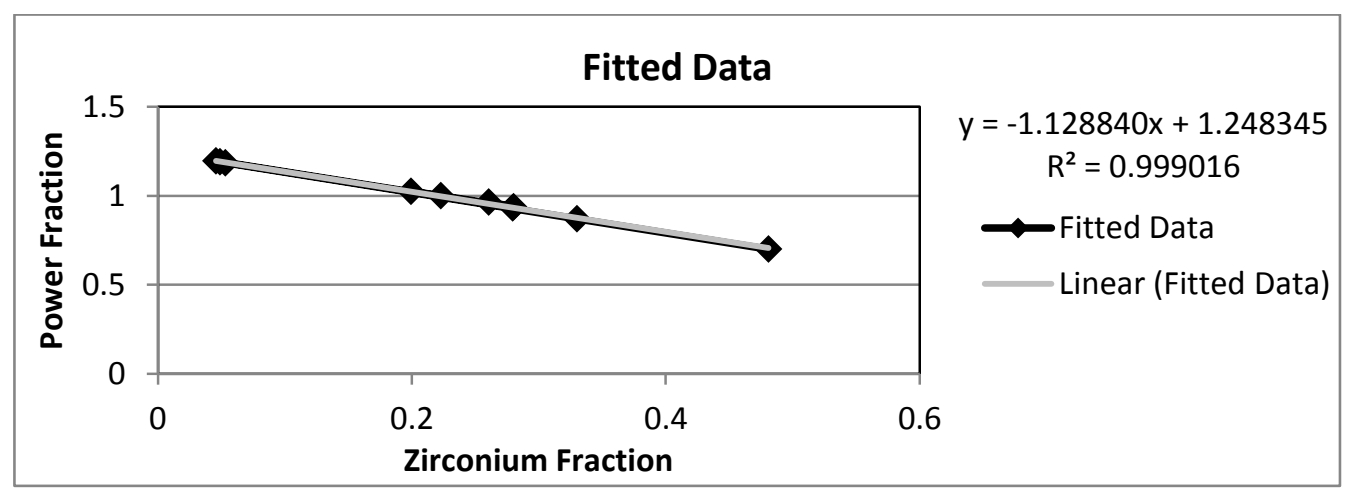

Figure 12: Functional fit of power fraction as a function of zirconium concentration averaged over both pin pitch simulations 


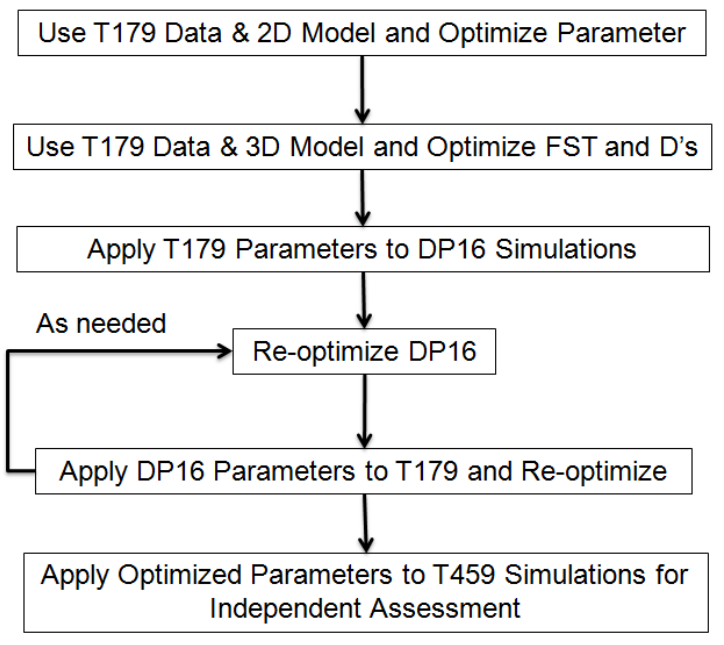

Figure 13: Iterative process for parameter determination and assessment

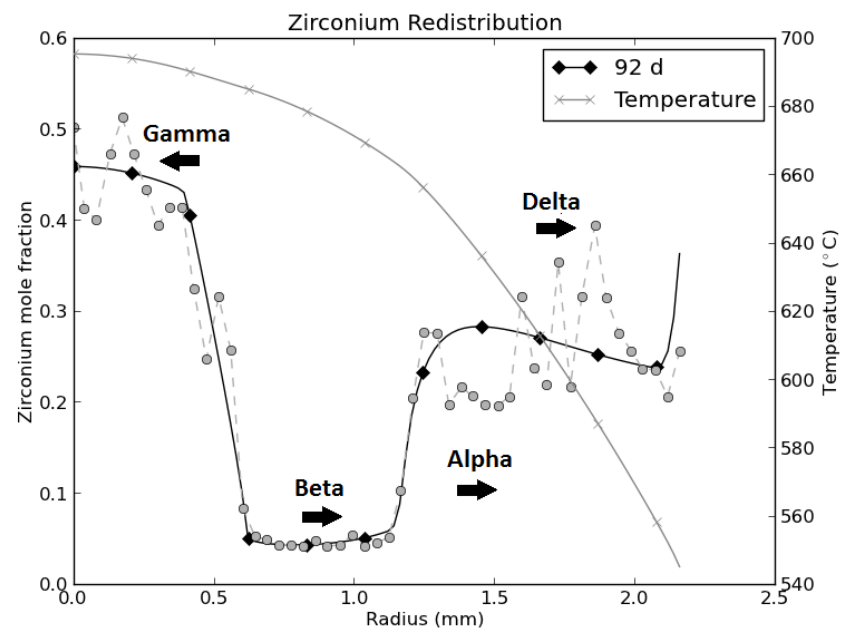

Figure 14: Phase mobility directions due to sign on material heat of transport 


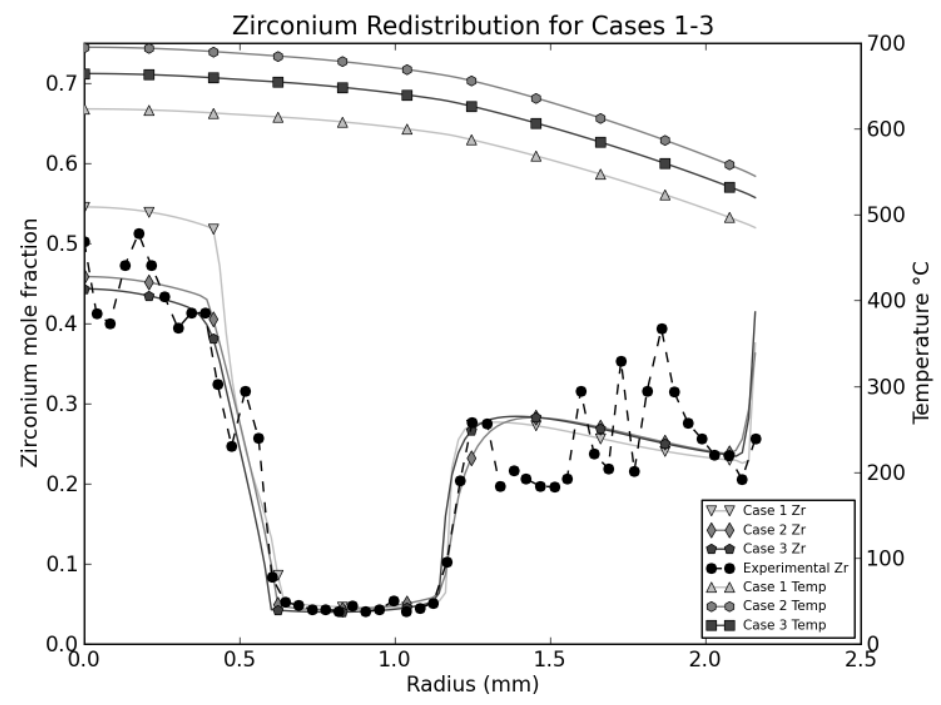

Figure 15: Case 1: adjust power and fuel surface temperature, Case 2: adjust transition temperatures and Case 3: adjust power, fuel surface temperature and transition temperatures

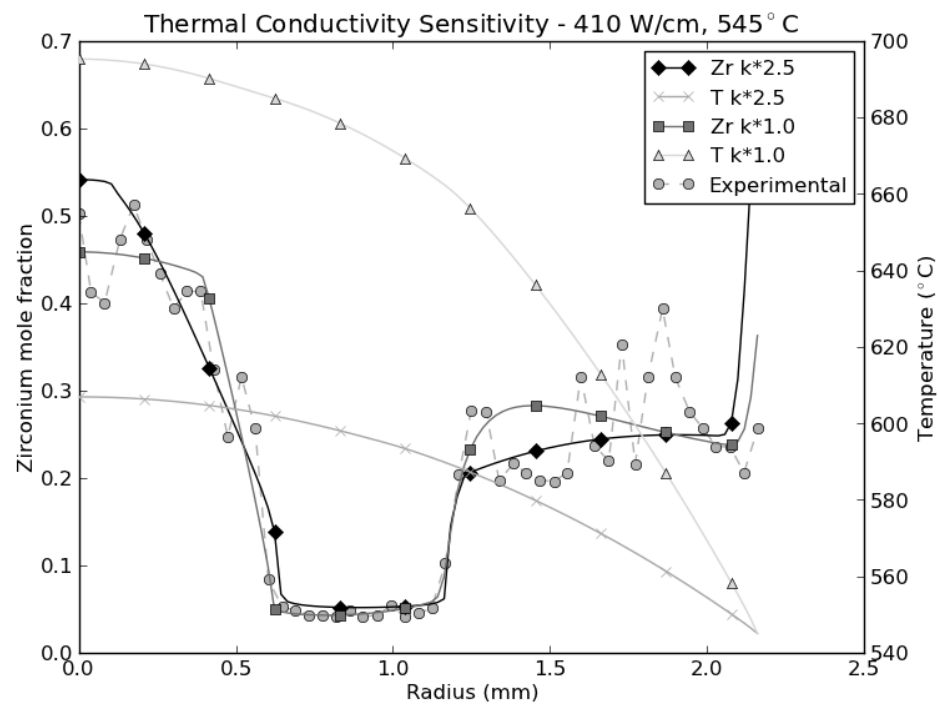

Figure 16: T179, match experimental data with thermal conductivity and diffusion coefficient modification only 


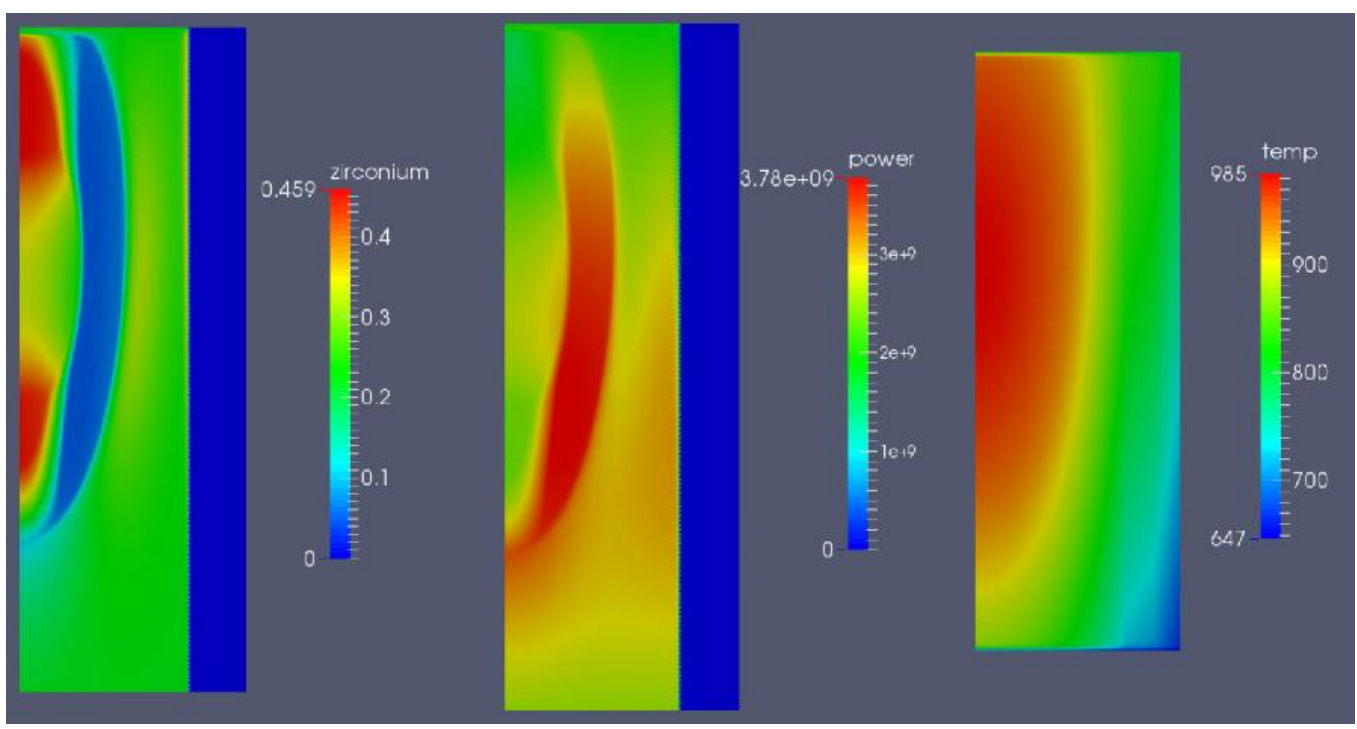

Figure 17: T179 full length 2D-RZ simulation, modeling parameters based on case 2 values, Zirconium concentration (mole fraction), power (\# fissions/sec), temperature (K)

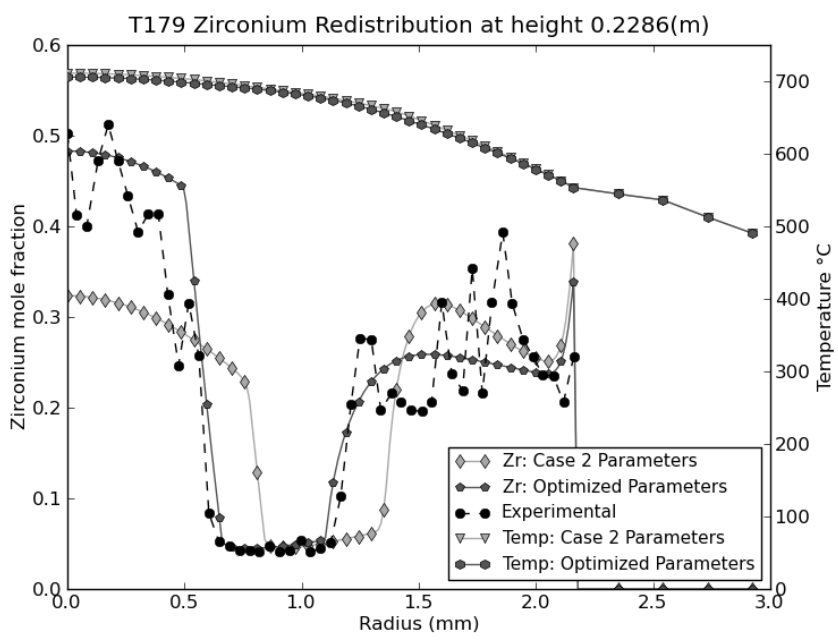

Figure 18: T179 full length $2 D-R Z$ results at $0.67 \mathrm{z} / \mathrm{L}$ with case 2 and optimized parameter simulation 


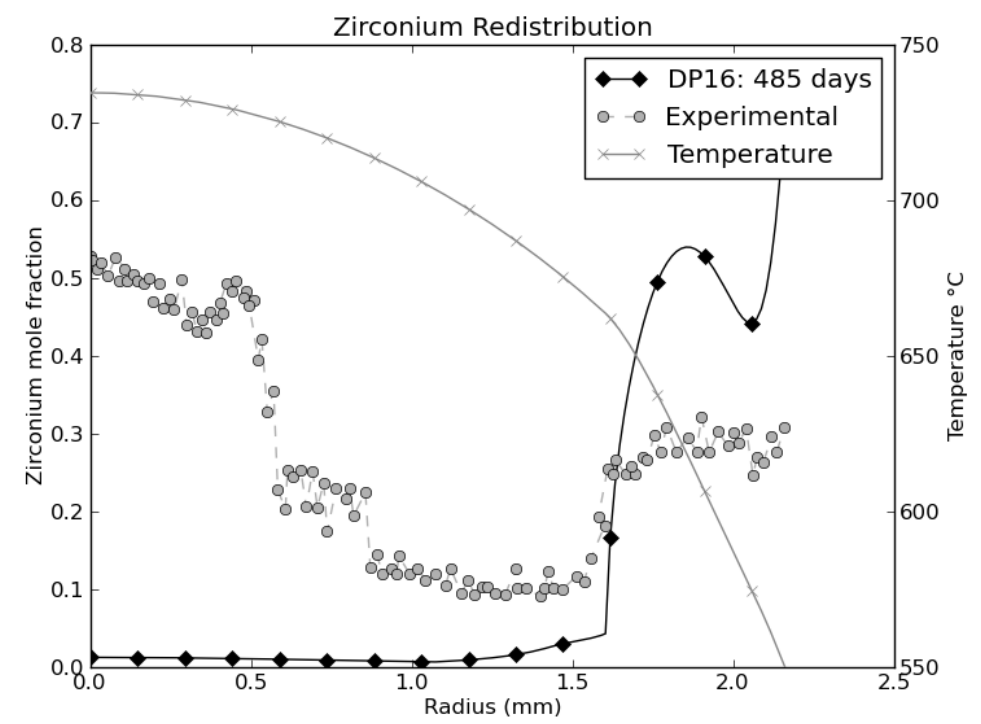

Figure 19: DP16 simulation with T179 optimized parameters

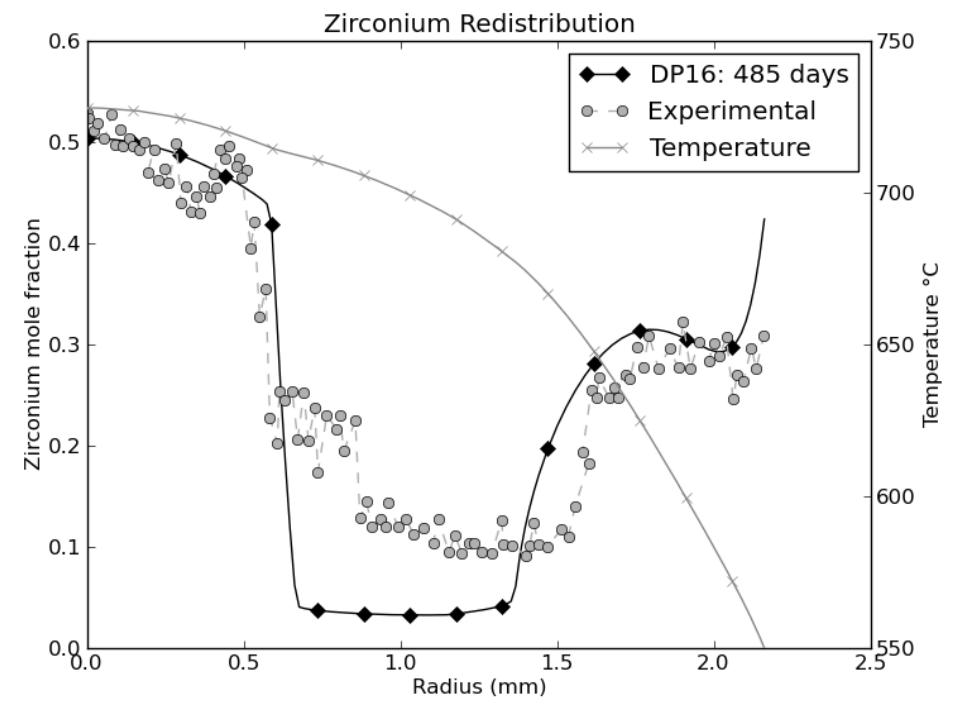

Figure 20: Final DP16 simulation with optimized parameters 


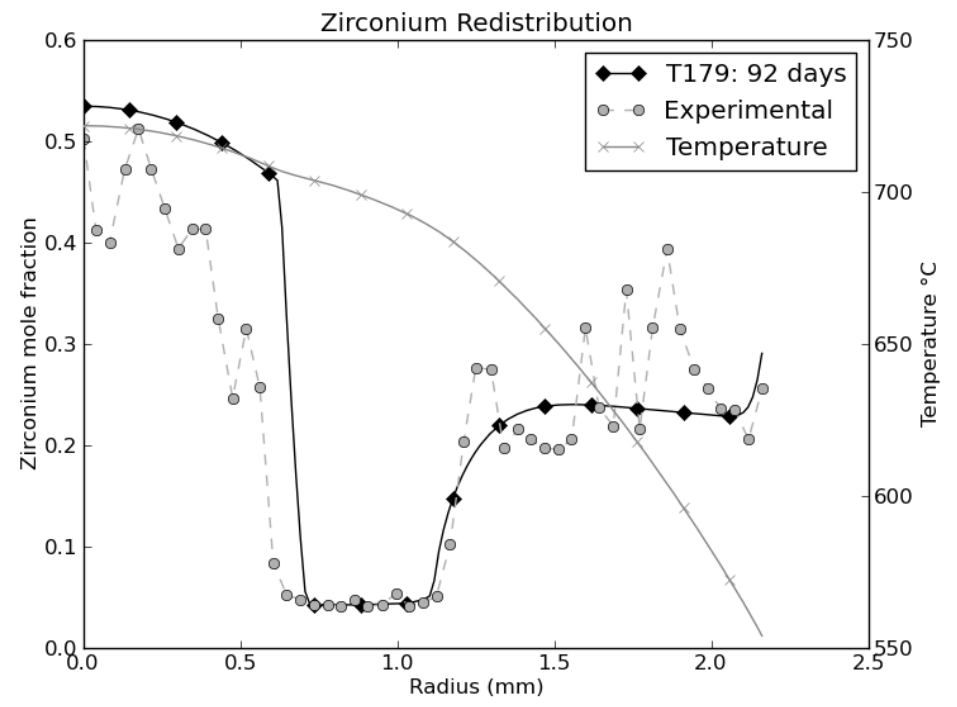

Figure 21: Final T179 simulation with optimized parameters
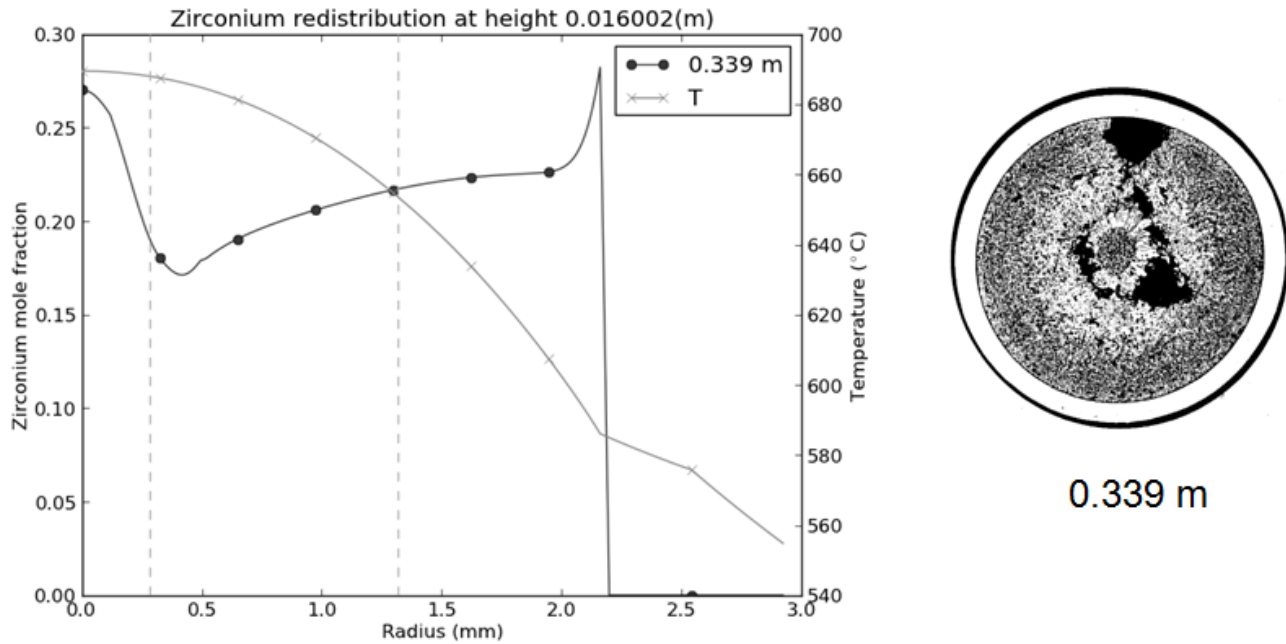

$0.339 \mathrm{~m}$

Figure 22: T459 rod, top results. The vertical dashed lines represent estimated phase transition locations derived from the picture. 

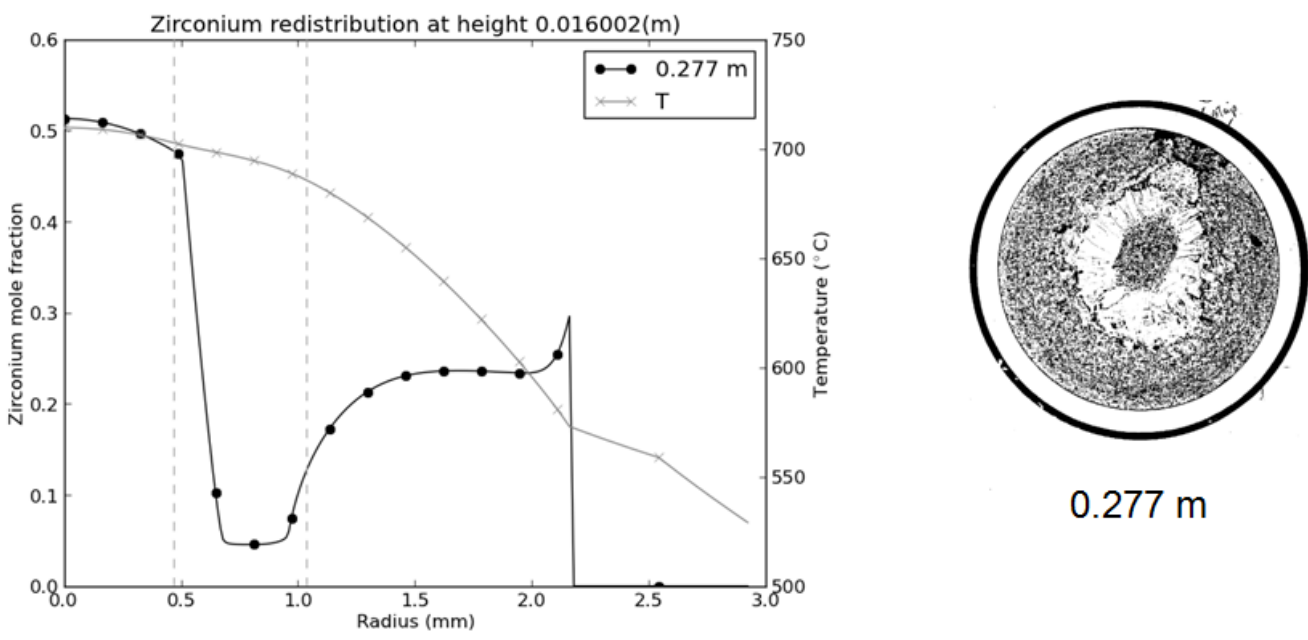

$0.277 \mathrm{~m}$

Figure 23: T459 rod, 2nd from top results. The vertical dashed lines represent estimated phase transition locations derived from the picture.
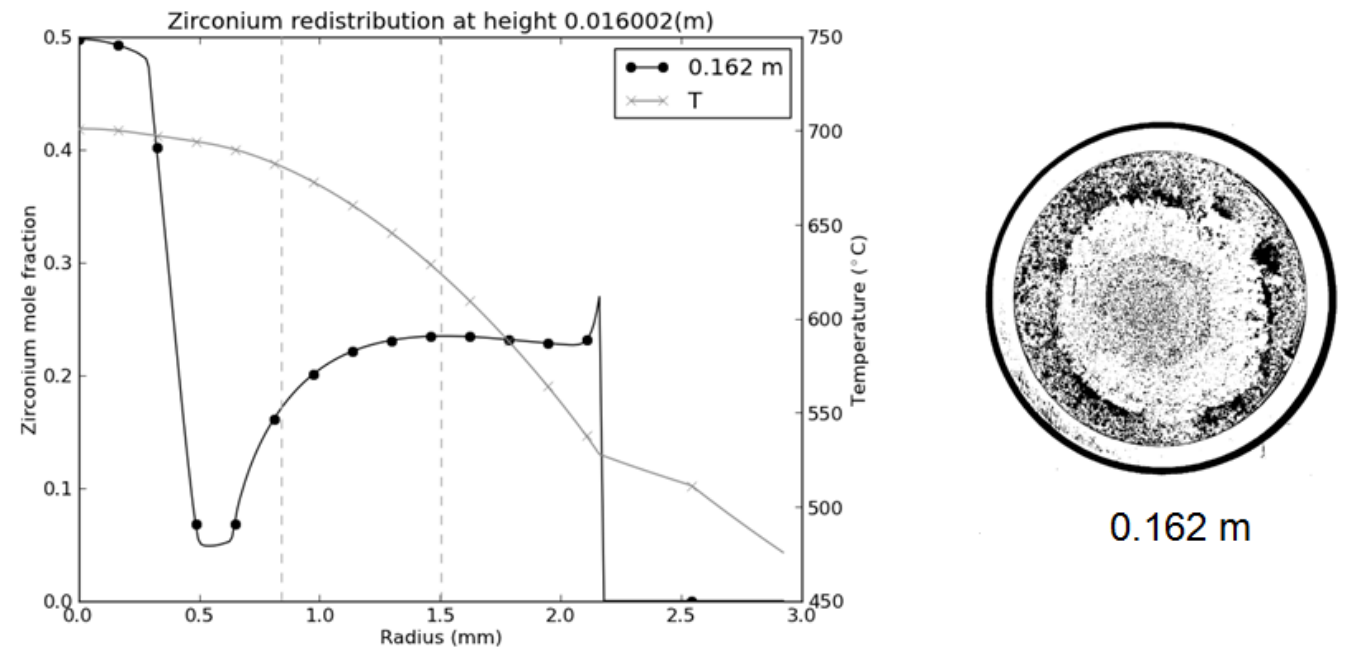

$0.162 \mathrm{~m}$

Figure 24: T459 rod, middle results. The vertical dashed lines represent estimated phase transition locations derived from the picture. 


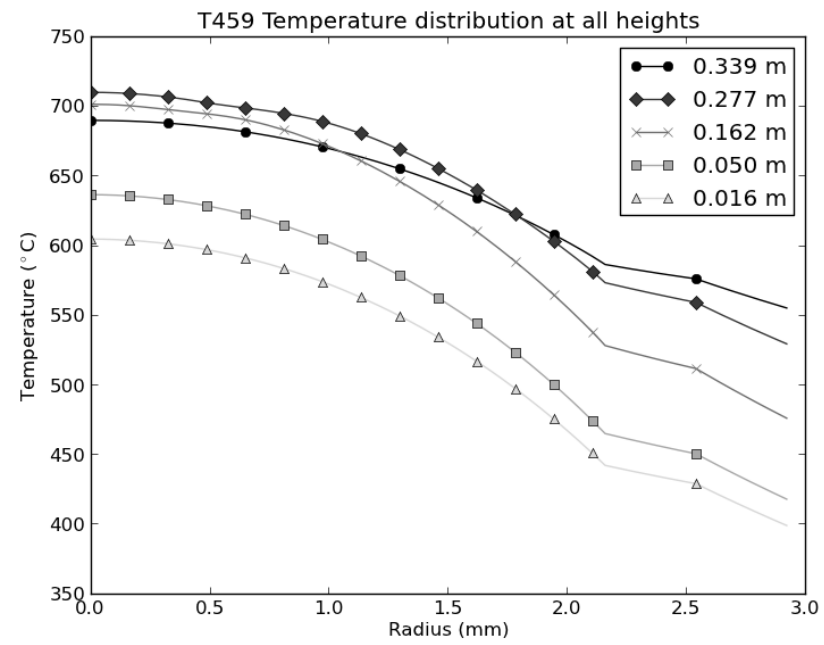

Figure 25: T459 temperature distribution at 5 axial levels

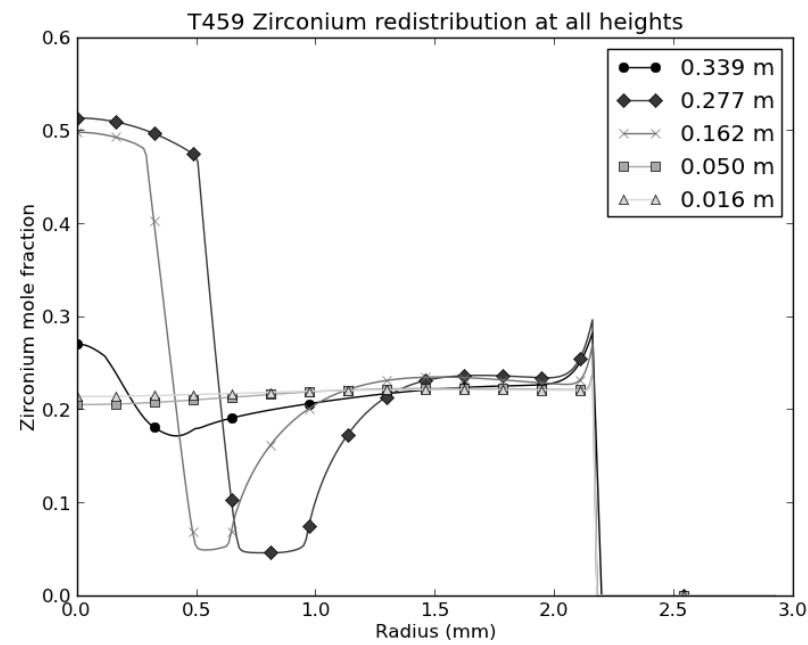

Figure 26: T459 zirconium distribution at 5 axial levels 